\title{
Noninvasive gene delivery to foveal cones for vision restoration
}

\author{
Hanen Khabou, ${ }^{1}$ Marcela Garita-Hernandez, ${ }^{1}$ Antoine Chaffiol, ${ }^{1}$ Sacha Reichman, ${ }^{1}$ Céline Jaillard, ${ }^{1}$ \\ Elena Brazhnikova, ${ }^{1}$ Stéphane Bertin,, ${ }^{1,2}$ Valérie Forster, ${ }^{1}$ Mélissa Desrosiers, ${ }^{1}$ Céline Winckler, ${ }^{1}$ \\ Olivier Goureau, ${ }^{1}$ Serge Picaud, ${ }^{1}$ Jens Duebel, ${ }^{1}$ José-Alain Sahel, ${ }^{1,2,3,4}$ and Deniz Dalkara' \\ 1Sorbonne Universités, UPMC Univ Paris 06, INSERM, CNRS, Institut de la Vision, Paris, France. ${ }^{2}$ CHNO des Quinze-Vingts, \\ DHU Sight Restore, INSERM-DCOS CIC 1423, Paris, France. ${ }^{3}$ Fondation Ophtalmologique Rothschild, Paris, France. \\ ${ }^{4}$ Department of Ophthalmology, The University of Pittsburgh School of Medicine, Pittsburgh, Pennsylvania, USA.
}

Conflict of interest: HK, DD, JD, and JAS are inventors on pending patent applications on noninvasive methods to target cone photoreceptors (EP17306429.6 and EP17306430.4). $\mathrm{MCH}, \mathrm{AC}, \mathrm{DD}, \mathrm{JD}$, and JAS are inventors on a pending patent on the use of iPSC to treat retinal degeneration (EP16306225). DD is an inventor on a patent of adeno-associated virus virions with variant capsid and methods of use thereof with royalties paid to Avalanche Biotech (W02012145601 A2). SR, OG, and JAS are inventors on a patent on $\mathrm{PSSC}$ retinal differentiation (W0 2014174492 A1). JAS is a founder and consultant for Pixium Vision and CenSight Biologics and is a consultant for Sanofi-Fovea, Genesignal, and Vision Medicines. SP is a founder of CenSight Biologics and a consultant for Pixium Vision. DD and JD are consultants for CenSight Biologics.

Submitted: July 5, 2017

Accepted: December 12, 2017

Published: January 25, 2018

Reference information:

JCI Insight. 2018;3(2): e96029.

https://doi.org/10.1172/jci.

insight. 96029.
Intraocular injection of adeno-associated viral (AAV) vectors has been an evident route for delivering gene drugs into the retina. However, gaps in our understanding of AAV transduction patterns within the anatomically unique environments of the subretinal and intravitreal space of the primate eye impeded the establishment of noninvasive and efficient gene delivery to foveal cones in the clinic. Here, we establish new vector-promoter combinations to overcome the limitations associated with AAV-mediated cone transduction in the fovea with supporting studies in mouse models, human induced pluripotent stem cell-derived organoids, postmortem human retinal explants, and living macaques. We show that an AAV9 variant provides efficient foveal cone transduction when injected into the subretinal space several millimeters away from the fovea, without detaching this delicate region. An engineered AAV2 variant provides gene delivery to foveal cones with a well-tolerated dose administered intravitreally. Both delivery modalities rely on a cone-specific promoter and result in high-level transgene expression compatible with optogenetic vision restoration. The model systems described here provide insight into the behavior of AAV vectors across species to obtain safety and efficacy needed for gene therapy in neurodegenerative disorders.

\section{Introduction}

The fovea - located at the center of the macula - is a specialized region of the retina that dominates the visual perception of primates by providing high-acuity color vision (1). The highest density of cones is found at the center of the fovea ( $<0.3 \mathrm{~mm}$ from the foveal center), devoid of rod photoreceptors (2). Cone density decreases by up to 100 -fold with distance from the fovea (3). Cone cells in the fovea are the primary targets of gene therapies aiming to treat inherited retinal diseases like midstage retinitis pigmentosa (4, 5) and achromatopsia (6). Currently, viral vectors encoding therapeutic proteins need to be injected into the subretinal space between the photoreceptors and the retinal pigment epithelium (RPE) cells in order to provide gene delivery to cones. In this approach, gene delivery is limited to cells that contact the local bleb of injected fluid. Furthermore, retinal detachment that occurs during subretinal injections is a concern in eyes with retinal degeneration. The earliest clinical trials using subretinal delivery of adeno-associated virus (AAV) to deliver a healthy retinal pigment epithelium-specific 65 kilodalton protein (RPE65) gene in Leber's congenital amaurosis patients (7-9) led to some improvements in vision, despite the detachment of the macula to deliver the viral vector $(10,11)$. However, the treatment was, in certain cases, complicated by macular holes and increased macular thinning in the case of subfoveal injections (11). Furthermore, contrary to the surrounding regions, there were no treatment benefits in the fovea (12). Gene therapy using AAV has also been studied for patients with choroideremia in which the macula was the target for gene delivery (13). The 6-month follow-up results from this latter study thus far suggest that subfoveal retinal detachment does not cause vision reduction in this region, but one of the patients in this trial had visual acuity loss in the treated eye compared with his untreated eye (13). With more gene therapies reaching clinical stages of application, there is a growing need to find new methods for delivering gene therapy to the fovea without detaching this brittle region (14). This can be achieved by engineering the viral vectors to permit gene delivery away from the injection site. AAV capsids that can provide gene delivery to foveal cones 
after injection into the vitreous cavity are one possible option. Another option would be through subretinal injections in the periphery using vectors that spread laterally to reach the foveal region.

To minimize the risks associated with foveal detachments, we developed vector-promoter combinations that can successfully deliver genes into foveal cones without detaching them from their underlying epithelium. As a key element to gene therapy, we first searched for promoters that are able to afford high-level transgene expression in cones without leading to off-target expression in neighboring cells. We then combined this promoter with 2 potent AAV capsid variants that are able to provide gene delivery to cone photoreceptors when injected intravitreally or via distal subretinal injections outside the fovea. We validated the utility of these gene delivery strategies in the context of cone-directed optogenetic therapy (15) using electrophysiology, histology, in vivo, and ex vivo imaging techniques in mouse, macaque, and human retinal tissue. Our results highlight the importance of viral vector development in overcoming surgical delivery challenges, as gene therapy to restore vision becomes a potentially attainable goal for those who treat inherited retinal degenerations in the clinic.

\section{Results}

Selection of a strong and specific cone cell-specific promoter in murine models. In order to find vector-promoter combinations suitable for strong and specific cone targeting away from the injection site, we compared several AAVs after intravitreal and subretinal delivery in mouse retinas. To enable efficient cone photoreceptor targeting, we used an engineered AAV variant called AAV2-7m8, which has been shown to target photoreceptors efficiently via both administration routes $(16,17)$. Specific targeting of cone cells has never been attempted using vitreally administered AAV. In order to find suitable promoter sequences for restricted gene expression in cones applicable in the clinic, we focused on promoters that have previously been validated in either nonhuman primate (NHP) (18) or human tissue (4). We generated AAV2-7m8 vectors encoding GFP under the control of mouse cone arrestin (mCAR), PR2.1 and PR1.7 promoters (synthetic promoters based on the human red opsin gene enhancer and promoter sequences - their size is equal to 2.1 and 1.7 kilobases, respectively) and injected them at equal titers into eyes of 6-week-old WT mice. Three weeks after subretinal injections, retinal cross-sections were stained with cone arrestin, and GFP expression was examined (Figure 1, A-C). We found GFP expression in both rod and cone photoreceptors with mCAR promoter, while PR2.1 and PR1.7 led to strong expression mostly in cones, as reported previously $(18,19)$. Using the same vectors, we obtained strikingly different expression patterns after intravitreal delivery (Figure 1, D-F). The mCAR promoter led to GFP expression in some cones but was leaky toward rods as well as cells of the inner nuclear layer (INL) and ganglion cell layer (GCL) (Figure 1D). Both PR2.1 and PR1.7 promoters led to more cone labeling than the mCAR promoter (Figure 1, D-F, and Supplemental Figure 1; supplemental material available online with this article; https://doi.org/10.1172/jci.insight.96029DS1). PR2.1 transduced more cones than PR1.7, but it also produced nonspecific GFP expression in the INL and GCL. Only the PR1.7 promoter showed GFP expression in cones with minimal expression in rods and was not leaky toward the inner retina (Figure 1F). Finally, as retinal disease state can influence AAV-mediated gene delivery and transgene expression patterns $(20,21)$, we validated AAV2-7m8-PR1.7 vector-promoter combination in a mouse model of retinal degeneration. We injected AAV2-7m8-PR1.7-GFP intravitreally in the retinal degeneration 10 (rd10) mouse model of retinitis pigmentosa. Two months after injection, GFP expression was restricted to cones (Figure $1 \mathrm{G}$ and Supplemental Figure 2). Based on these results, we decided to test it in the primate retina.

Bioinformatic analysis of $m C A R, P R 1.7$, and PR2.1 promoter sequences. Before moving on to further studies in other species, we aimed to better understand the reasons behind the divergent expression patterns obtained with the 3 promoters. To do so, we analyzed transcription factor (TF) binding sites within each promoter sequence using bioinformatics (Supplemental Tables 1 and 2). The present analysis aimed to answer the following questions: (i) why is PR1.7 more efficient than PR2.1 in cones (18), and (ii) why do PR2.1 and mCAR promoters lead to off-target expression after intravitreal administration? We hypothesized that the differential expression patterns observed between PR1.7 and PR2.1 are due to additional TF binding sites found in the 337-bp sequence located in the $5^{\prime}$ region of the PR2.1 promoter but not in the PR1.7 promoter (Figure 2, A and B). Interestingly, we found a chicken ovalbumin upstream promoter-transcription factor I (COUP-TFI) binding site within this 337-bp sequence (Supplemental Table 1). COUP-TFI has been shown to suppress green opsin gene (OpnImw) expression in the mouse retina (22) and might thus be accountable for lower expression with the PR2.1 promoter in macaque cones when AAV is delivered subretinally as previously shown (18). Within the same specific 337-bp region, we also found 

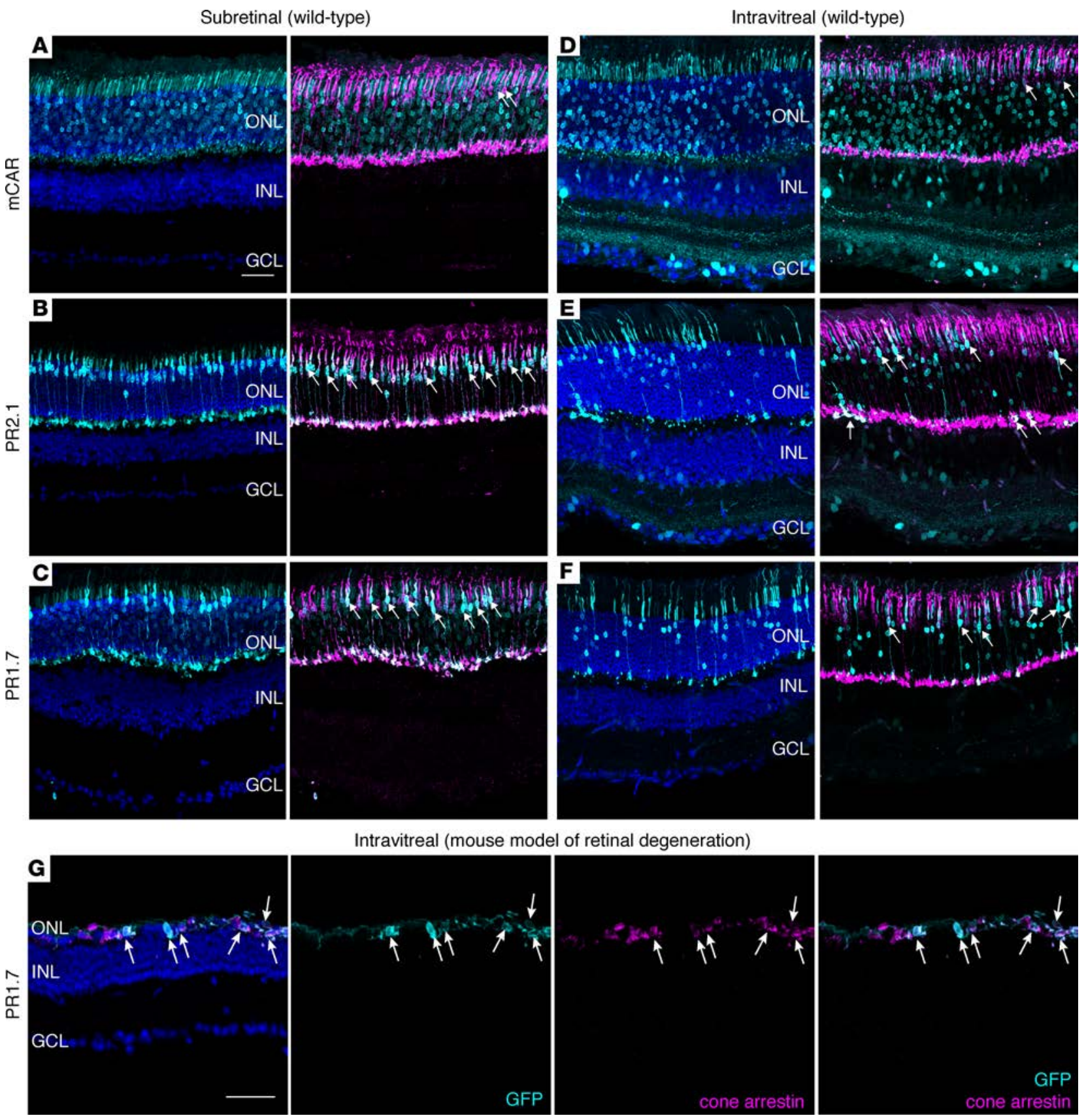

Figure 1. Adeno-associated viral (AAV) vector administration route defines retinal transduction patterns with mCAR, PR2.1, and PR1.7 promoters. (A-C) Representative retinal cross sections of WT mouse retinas ( $n=6$ eyes per condition) 3 weeks after subretinal injection of AAV2-7m8-mCAR-GFP (A), AAV2-7m8-PR2.1-GFP (B), and AAV2-7m8-PR1.7-GFP (C). (D-F) Representative retinal cross sections 2 months after intravitreal injection ( $n=6$ eyes per condition) of AAV2-7m8-mCAR-GFP (D), AAV2-7m8-PR2.1-GFP (E), and AAV2-7m8-PR1.7-GFP (F). Scale bar: $50 \mu \mathrm{m}$ in A-F. (G) GFP expression in rd10 retina ( $n=4$ eyes) 2 months after intravitreal injection of AAV2-7m8-PR1.7-GFP. Transduced cone cell bodies remaining after degeneration express GFP (cyan). Cone arrestin is shown in magenta, and DAPI is shown in blue. Native GFP expression is shown in cyan, and arrows indicate cells where cone arrestin is colocalized with GFP. Scale bar is $50 \mu \mathrm{m}$ in G. mCAR, mouse cone arrestin promoter; PR1.7 and PR2.1, promoters of 1.7 and 2.1 kilobases in length, respectively, based on the human red opsin gene enhancer and promoter sequences.

multiple binding sites for generic, ubiquitous activator TFs (Figure 2B and Supplemental Table 1), such as CCAAT/enhancer binding protein $\beta$ (CEBPB) and general transcription factor II-I (GTF2I). These additional binding sites of TFs that enhance binding and basal transcriptional machinery assembly and that are not specifically expressed in cones might be responsible for some of the off-target expression observed with PR2.1 compared with PR1.7 (Figure 2). We also analyzed TF binding sites in the genomic mouse Arr3 promoter sequence to explain the lack of specificity using the short version of this promoter (referred to as 
A

Human red opsin gene expression

In the genome
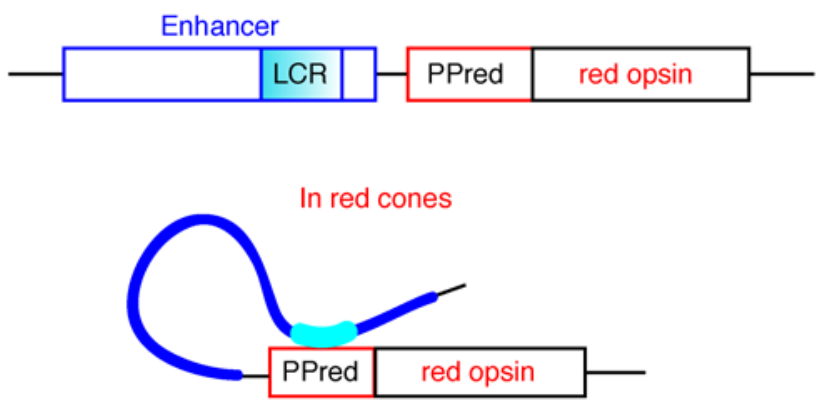

chromosomal loop favors red opsin gene expression
B
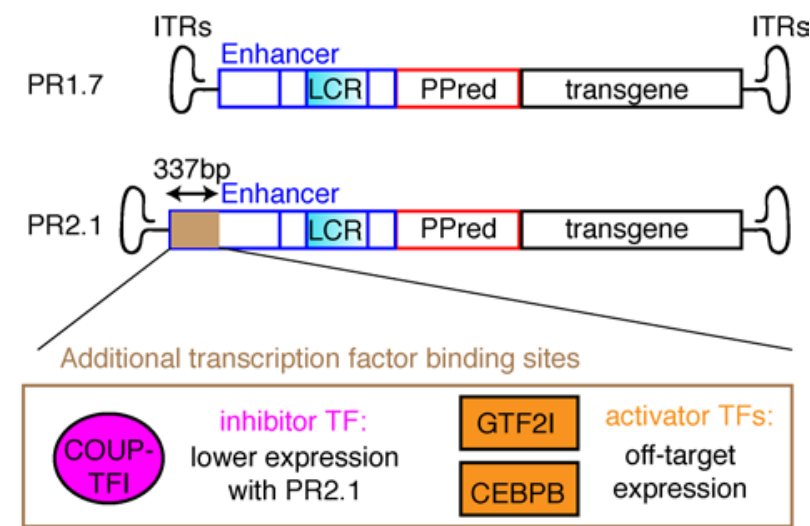

AAV vectors with the synthethic human red opsin promoters pR2.1 and pR1.7

Rs
C

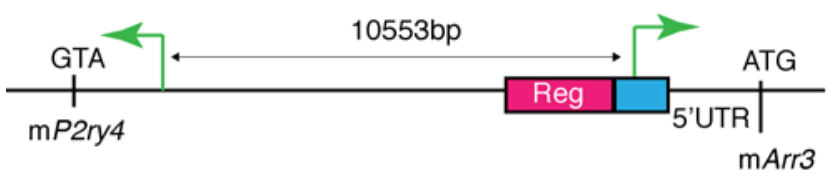

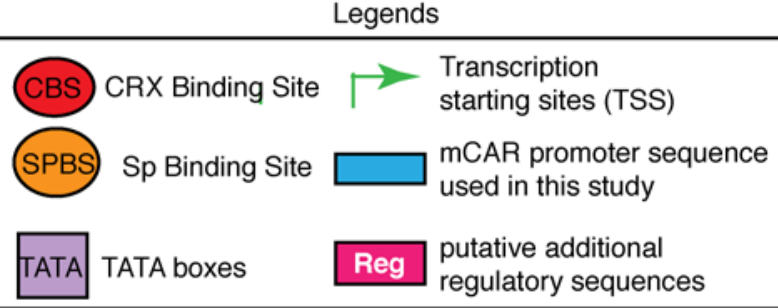

E

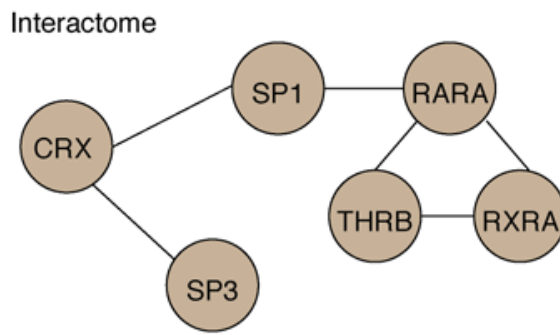

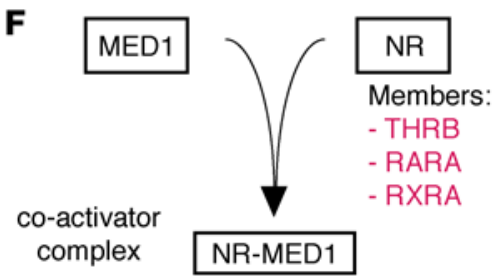

Function: cell- or tissue-specific regulation of gene transcription

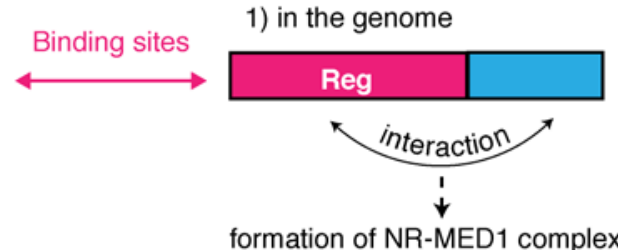

$\rightarrow$ cone-restricted expression
2) with mCAR-GFP vector

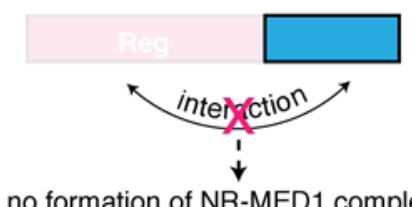

no formation of NR-MED1 complex $\rightarrow$ loss of cone-restricted expression

Figure 2. Model for the regulation of transgene expression under the control of PR2.1 and PR1.7 synthetic promoters and mouse cone arrestin (mCAR) promoter. (A) Red opsin gene is located on the $\mathrm{X}$ chromosome. It has its own proximal promoter and shares its enhancer sequence with green opsin gene. Chromosomal loops between the enhancer and the red opsin proximal promoter provide cell-type specificity of gene expression. PPred, proximal promoter of red opsin gene; LCR, locus control region. (B) Schematic representation of PR2.1 and PR1.7 promoter constructs. Interaction with inhibitory transcription factors such as COUP-TFI (chicken ovalbumin upstream promoter-transcription factor), that binds the 337bp region specific to PR2.1 might explain low expression levels obtained with PR2.1 compared with PR1.7 in macaque cones subretinally (18). On the other hand, activator TFs such as CEBPB (CCAAT/enhancer binding protein $\beta$ ) and GTF2I (general transcription factor II-I) that are not specific to cones likely lead to off-target expression in other retinal cells when injected into the vitreous. ITRs, inverted terminal repeats. (C) Structure of cone arrestin 3 genomic locus region. Transcription starting sites (TSS) of mouse arrestin 3 (mArr 3 ) gene and mouse pyrimidinergic receptor P2Y4 (mP2ry4) gene are separated by 10.5 kilobases. The short 521-bp mCAR promoter used in this study is shown in blue and the supposed regulatory region referred to as "Reg" in magenta. (D) Structure of the 521-bp mCAR promoter portion used in this study. This sequence contains CRX-binding sites (CBS) and SP (Specificity Protein) binding sites. It also contains 1 TATA and 1 TATA-like box. (E) Interactome network of several transcription factors that bind cone arrestin genomic promoter analyzed using the STRING tool. CRX (cone-rod homeobox protein), SP, RARA (retinoic acid receptor $\alpha$ ), RXRA (retinoid X receptor $\alpha$ ), and THRB (thyroid hormone receptor $\beta$ 2). (F) NR-MED1 transcription regulator complex confers gene expression specificity. MED1 (mediator complex subunit 1) is a transcription activator when associated to nuclear receptors (NRs). RARA, RXRA, and THRB are NRs. Several NR binding sites for RARA, RXRA, and THRB were found in the Reg region. AAV, adeno-associated virus; mCAR, mouse cone arrestin promoter; PR1.7 and PR2.1, promoters of 1.7 and 2.1 kilobases in length, respectively, based on the human red opsin gene enhancer and promoter sequences. 
Table 1. Summary of injections in nonhuman primates

\begin{tabular}{|c|c|c|c|c|c|c|c|c|}
\hline Animal & Age (years) & Weight (kg) & Sex & Injection type; eye & AAV capsid & Transgene & Dose (vg/eye) & Volume $(\mu \mathrm{l})$ \\
\hline NHP 1 & 7 & 8.65 & $M$ & intravitreal; LE & AAV2-7m8 & PR1.7-GFP & $1 \times 10^{11} \mathrm{vg}$ & 100 \\
\hline NHP 2 & 8 & 11.81 & M & intravitreal; LE & AAV2-7m8 & CMV-GFP & $1 \times 10^{11} \mathrm{vg}$ & 100 \\
\hline NHP 4 & 4 & 5.03 & M & intravitreal; RE & AAV2-7m8 & PR1.7-Jaws-GFP & $1 \times 10^{11} \mathrm{vg}$ & 100 \\
\hline NHP 5 & 4 & 6.26 & M & $\begin{array}{l}\text { subretinal, sup; RE } \\
\text { intravitreal; LE }\end{array}$ & $\begin{array}{l}\text { AAV9-7m8 } \\
\text { AAV2-7m8 }\end{array}$ & $\begin{array}{l}\text { PR1.7-Jaws-GFP } \\
\text { PR1.7-Jaws-GFP }\end{array}$ & $\begin{array}{l}1 \times 10^{10} \mathrm{vg} \\
1 \times 10^{10} \mathrm{vg}\end{array}$ & $\begin{array}{l}50 \\
50\end{array}$ \\
\hline NHP 7 & 3 & 3 & M & subretinal, inf; RE & AAV9-7m8 & PR1.7-Jaws-GFP & $5 \times 10^{9} \mathrm{vg}$ & 50 \\
\hline NHP 8 & 3 & 3.23 & M & subretinal, inf; RE & AAV9-7m8 & PR1.7-Jaws-GFP & $5 \times 10^{9} \mathrm{vg}$ & 50 \\
\hline
\end{tabular}

NHP, nonhuman primate; kg, kilograms; M, male; sup, superior bleb; inf, inferior bleb; LE, left eye; RE, right eye; AAV, adeno-associated virus; CMV, cytomegalovirus promoter; PR1.7, Promoter 1.7 kilobases, based on human red opsin gene enhancer and promoter sequences; vg, viral genome.

mCAR) used in the AAV constructs. The short sequence consists of a 521-bp portion of the genomic proximal CAR promoter (Figure 2, C and D) and presents a TATA-box, a TATA-like box, as well as binding sites for cone-rod homeobox protein (CRX) and specificity protein (SP) TFs (23) (Figure 2D). However, the "Reg" sequence (Figure 2C) modulating Arr3 promoter activity (23) located directly upstream of the 521-bp region is excluded from the short mCAR sequence (Figure $2 \mathrm{C}$ ). Based on the interactome of the TFs binding mCAR promoter obtained from the STRING database (24), CRX and SP TFs interact with each other and with retinoic acid receptor $\alpha$ (RARA), retinoid $X$ receptor $\alpha$ (RXRA), and thyroid hormone receptor $\beta$ (THRB) TFs (Figure 2E). These 3 nuclear receptors (NRs) are involved in cell type-specific regulation of gene expression via mediator complex subunit 1 (MED1) (25) by forming a cell-specific transcription coactivator complex $(26,27)$ (Figure $2 \mathrm{~F}$ ). CRX and SP binding sites are located on the 521-bp region, while RXRA, RARA, and THRB binding sites are positioned on the Reg region (Figure 2F). Moreover, the Reg region contains 5 binding sites for THRB2, an important NR expressed in cones (28) (Supplemental Table 2). For all of these reasons, removal of the Reg region is likely responsible for the off-target expression observed with the short mCAR promoter (Figure 2F).

Safe gene delivery to macaque foveal cones via intravitreal administration of $A A V$. We and others have shown transduction of macaque cones using AAV variants with ubiquitous promoters (16, 29-32), but achieving cone transduction by vitreally administered AAV has only been possible at high doses, leading to inflammation $(16,29)$. We reasoned that foveal cone targeting could be achieved if we use a strong cone-specific promoter at lower intravitreally injected AAV doses compatible with safety $(29,33)$. To test if such "dose sparing" is possible, we injected 2 macaque eyes with AAV2-7m8-PR1.7-GFP and 2 other macaque eyes were injected with AAV2-7m8-GFP under the control of the cytomegalovirus (CMV) promoter at a dose of $1 \times 10^{11}$ viral genomes (vg) per eye (Table 1). Using in vivo eye fundus imaging, we observed GFP expression as early as 2 weeks after injection with CMV and increased until 2 months after injection (Figure 3, A and B, and Supplemental Figure 3). GFP fluorescence was predominantly in the periphery and in the parafoveal region. GFP expression with PR1.7 became detectable 4-6 weeks after administration and was restricted to the fovea (Figure 3, C and D). There was no detectable damage to the fovea as assessed by optical coherence tomography (OCT) (Figure 4). We then examined flatmounts of the maculas and cryosections of the fovea using confocal microscopy, with equal acquisition settings for each eye (Figure 3). These images corroborated the in vivo findings showing specific and robust foveal cone transduction from the vitreous (Supplemental Video 1), at a dose of $1 \times 10^{11}$ particles, using AAV2-7m8-PR1.7. About 58\% of the hCAR ${ }^{+}$cells were found to express detectable levels of GFP in the foveola. The CMV promoter did not provide detectable transgene expression in cones at an identical dose.

Therapeutic gene delivery to foveal cones for vision restoration using optogenetics. We next aimed to evaluate the possibility of using this promoter for therapeutic gene delivery. There is no existing blind macaque or primate model of retinal degeneration to test functional outcomes after gene replacement (e.g., CNGB3 for treatment of achromatopsia). However, it is possible to evaluate vision restoration in WT macaques using optogenetic strategies, since we can distinguish between optogenetic-mediated light responses versus endog- 
enous cone opsin-mediated responses (4). We evaluated the potential of optogenetic vision restoration by expression of Jaws, a hyperpolarizing microbial opsin (15), in foveal cones. We injected 1 macaque eye with $1 \times 10^{11} \mathrm{vg}$ of AAV2-7m8-PR1.7-Jaws-GFP in the vitreous to evaluate its therapeutic potential for reactivation of dormant cones in midstage retinitis pigmentosa as described previously in mice $(4,15)$. We found high-level Jaws-GFP expression restricted to the foveal cones in the injected eye (Figure 5, A-C) similar to GFP expression alone (Figure 3). The animal was then sacrificed 2 months after injection, and half of the retina was processed for histology. Retinal flat-mounts showed typical anatomy of cones in the foveola (Figure 5, D and E), the region of the fovea that contains densely packed cones responsible for our high-acuity vision. Immunostaining for hCAR was used to quantify transduced cones (Supplemental Figure 4). About $50 \%$ of the $\mathrm{hCAR}^{+}$cells were found to express detectable levels of Jaws-GFP in this foveola.

The other half of the retina was conserved as explants (34) for characterization of optogenetic light responses arising from the hyperpolarizing pump Jaws (Figure 5, F-K). Electrophysiological recordings were performed on transduced cones expressing Jaws and in control cones without Jaws expression (Figure 5, F and G). Whole-cell patch-clamp recordings in $\mathrm{GFP}^{+}$Jaws cones exhibited robust light responses to orange light flashes $(n=4)$ (Figure $5, \mathrm{H}$ and I). Action spectrum of recorded cells showed that highest light responses were obtained using orange light between $575 \mathrm{~nm}$ and $600 \mathrm{~nm}$ (Figure 5J) as previously shown for Jaws (15). Jaws-expressing cones recorded in current-clamp configuration displayed light-elicited hyperpolarizations followed by short depolarizations ( $n=4$ cells), while control cones did not respond to the same light stimuli ( $n=3$ cells) (Figure $5 \mathrm{~K}$ ).

Finally, we injected intravitreally another macaque eye with $1 \times 10^{10}$ particles of AAV2-7m8-PR1.7Jaws-GFP to evaluate feasibility of foveal transduction at even lower doses. We obtained detectable foveal Jaws expression even with this lower dose, although expression levels were lower than with $1 \times 10^{11}$ particles (Supplemental Figure 5, A and B). Altogether, all 4 macaque eyes injected with AAV2-7m8-PR1.7GFP $(n=2)$ or Jaws-GFP $(n=2)$ show reproducibility and strength of the intravitreal approach compatible with optogenetic reactivation of cones.

Enhanced optogenetic responses in foveal cones via distal subretinal administration of AAV9-7m8-Jaws. Transduction of foveal cones via intravitreal injection of AAV2-7m8 with a strong cone promoter is likely an ideal approach to treat cones in fragile retinas of retinitis pigmentosa patients with dormant cones present mainly in the foveola. However, for achromatopsia patients, as well as the subset of retinitis pigmentosa patients with strong neutralizing antibody titers against AAV2 (33), a subretinal approach might be advantageous. Previous studies have shown that subretinal injection of AAV9 leads to efficient transgene expression in cones both centrally and peripherally at low doses, likely due to the abundance of galactosylated glycans, the primary receptor for AAV9, on cone photoreceptors $(30,35)$. Based on this, we reasoned that an enhanced AAV9 variant might afford efficient transduction of foveal cones from a distal bleb. We previously described a variant called AAV9-7m8, which provides a 30-fold increase in infectivity over AAV9 (17). To promote foveal cone gene delivery through a distal subretinal injection site, we used an AAV9-7m8 variant. We injected 1 animal subretinally with $5 \times 10^{10}$ particles of this vector encoding Jaws-GFP into the peripheral retina (Figure 6, A and B) without detaching the fovea. As early as 2 weeks after injection, we observed strong Jaws-GFP fluorescence in the bleb (delimited by the dashed cyan line) and also in the foveola (Figure 6C). Fluorescence intensity was higher in the foveola compared with intravitreally treated retinas. We observed the same result in a second eye injected with $1 \times 10^{10} \mathrm{vg}$ of the same vector (Supplemental Figure 5C). To further confirm that the superior peripheral blebs did not descend toward the fovea once the animal was in upright position and to see if further dose reduction was possible, we injected 2 other eyes with a dose of $5 \times 10^{9} \mathrm{vg}$, this time inferior to the fovea (Table 1). Using OCT, we observed that the fovea was not detached after surgery (Supplemental Figure 6). The same expression pattern, extending to the foveal cones, was obtained in these retinas (Supplemental Figure 5D). These results collectively show the reproducibility of this approach and its compatibility with low viral doses.

Flatmounts were then prepared, and fovea was processed for histology and showed strong JawsGFP expression in a large population of cones (Figure 6D) in the region between the injection site and inside the fovea (Figure 6, D-F). Cell counting of GFP and $\mathrm{CAR}^{+}$cells showed about $95 \%$ of cones were labeled using this subretinal approach (Figure 6, E and F) compared with about 50\% obtained with intravitreal injection (Supplemental Figure 4). The amplitude of photocurrents were 5 times higher in Jaws cones after subretinal delivery compared with those in Jaws cones after intravitreal delivery, with similarly shaped light sensitivity curves (Figure $5 \mathrm{H}$ and Figure $6, \mathrm{G}$ and $\mathrm{H}$ ). This is likely due to 

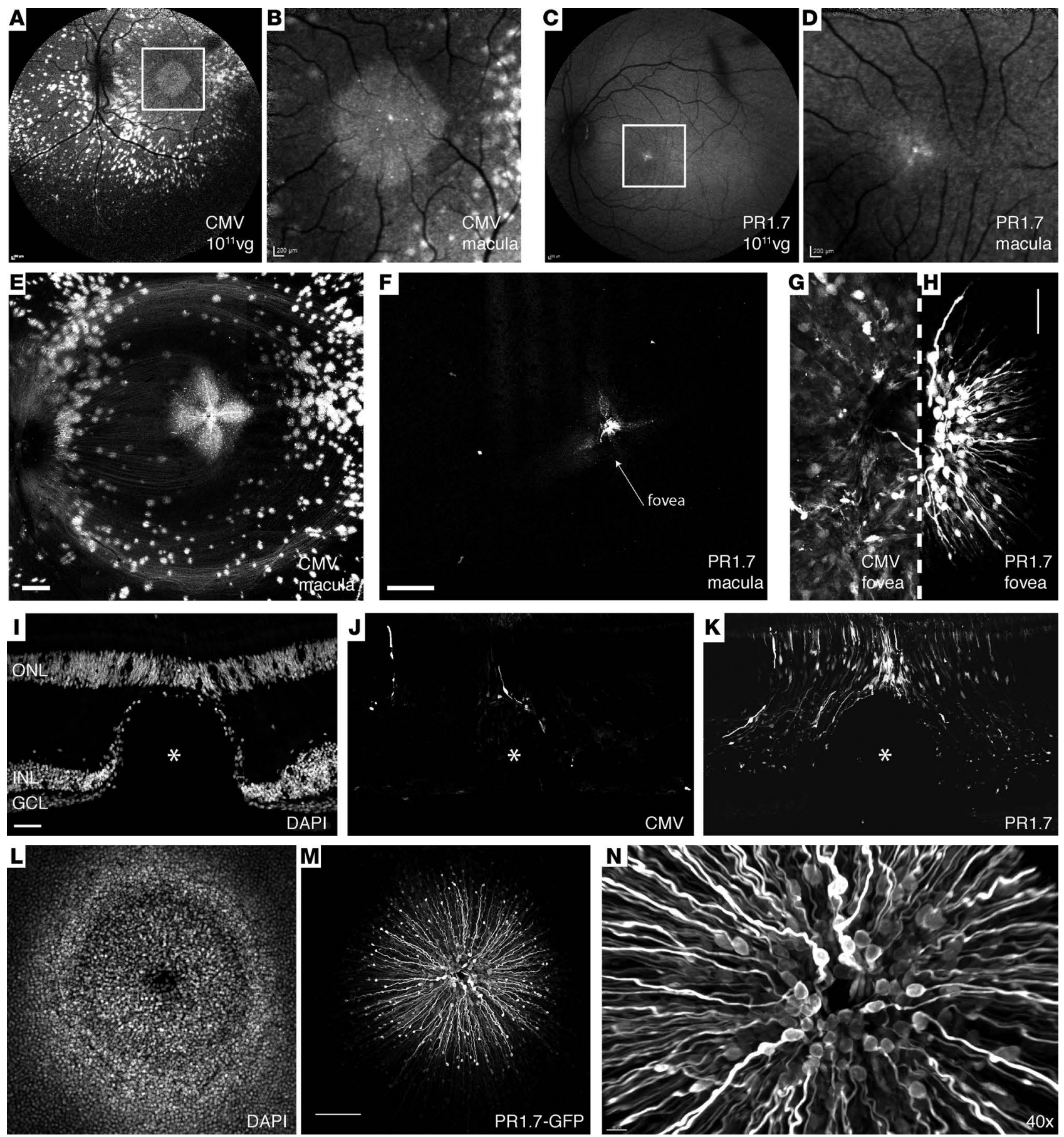

Figure 3. Foveal cone transduction with PR1.7 promoter versus cytomegalovirus (CMV) promoter 2-3 months after intravitreal delivery of AAV2-7m8 in nonhuman primates. Representative eye fundus images after intravitreal injection of AAV2-7m8-CMV-GFP ( $n=2$ eyes) (A and B) or AAV2-7m8-PR1.7-GFP $(n=2$ eyes) (C and $\mathbf{D})$ at $1 \times 10^{11}$ viral particles per eye. $\mathbf{B}$ and $\mathbf{D}$ are inset of images shown in $\mathbf{A}$ and $\mathbf{C}$. Scale bars: $200 \mu \mathrm{m}$. Confocal images of the maculas mounted with the ganglion cell layer facing upward using CMV (E) and PR1.7 (F) promoters. Scale bars: $500 \mu \mathrm{m}$. (G and $\mathbf{H})$ Zoomed images of the fovea with CMV (G) and with PR1.7 (H). Scale bars: $100 \mu \mathrm{m}$ in $\mathbf{G}$ and $\mathbf{H}$. (I-K) Retinal cryosections at the level of the fovea. (I) DAPI staining at the level of the fovea. Asterisk represents foveal pit. GFP expression under the control of CMV (J) or PR1.7 (K) promoters. Scale bar: $50 \mu \mathrm{m}$ in I, J, and K. (L-N) Confocal image projection of the whole foveal flatmount showing nuclei (L) and GFP expression in cones (M). Scale bar: 100 $\mu \mathrm{m}$. (N) Zoom into 3-D-reconstructed fovea seen in $\mathbf{M}$ with close-up to the cell bodies (facing upward). Scale bar: 10 $\mu \mathrm{m}$. AAV, adeno-associated virus; PR1.7, a promoter of 1.7 kilobases in length, based on the human red opsin gene enhancer and promoter sequences. 

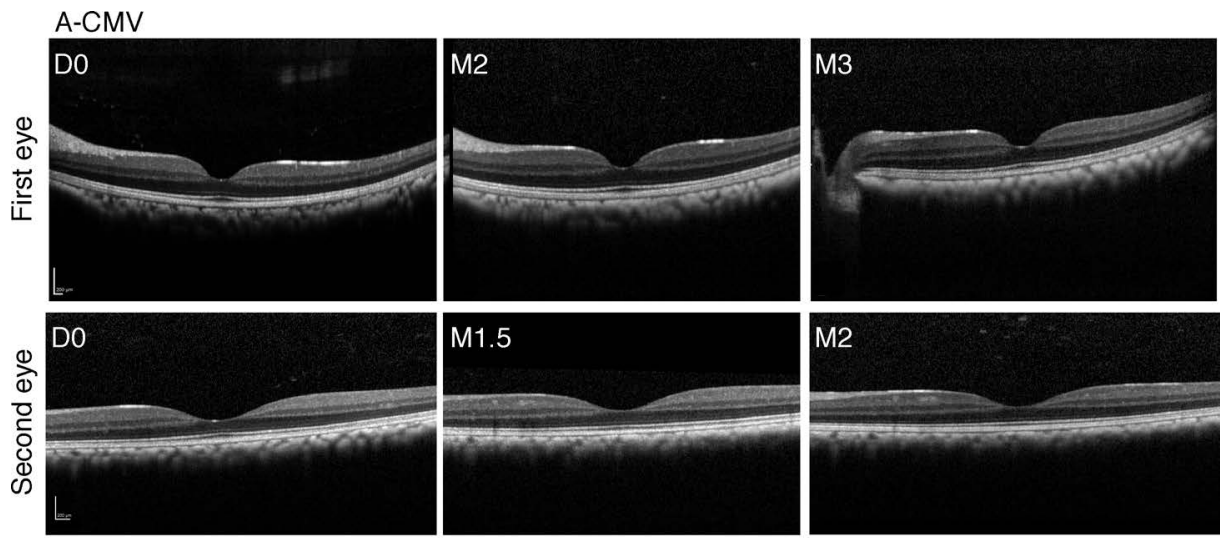

\section{B-PR1.7}
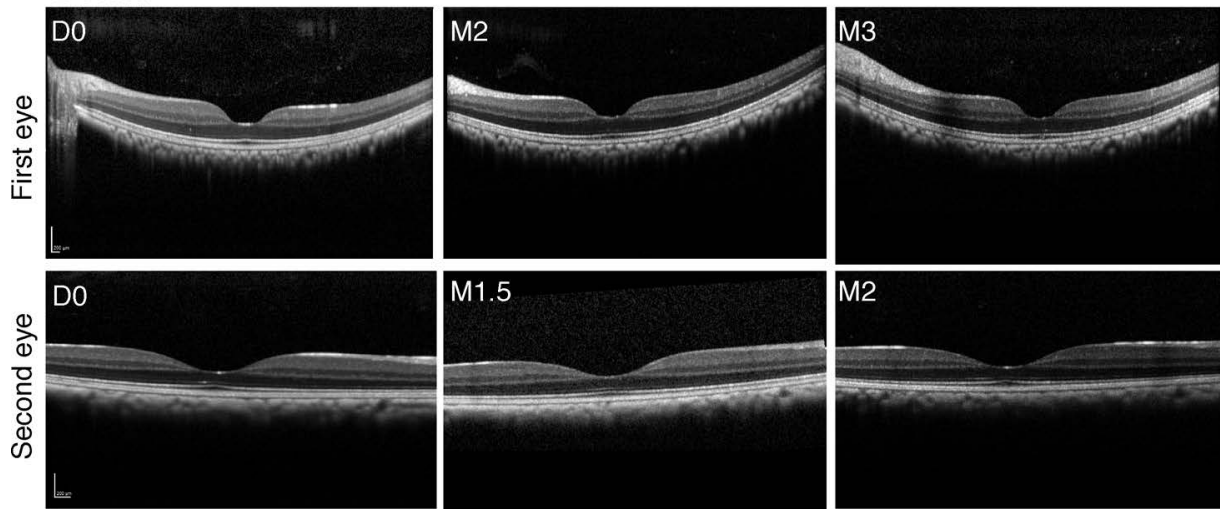

Figure 4. Optical coherence tomography (OCT) follow-up of AAV2-7m8-CMV-GFP- and PR1.7-GFP-treated eyes. (A) Foveal OCT images of CMV-treated eyes $(n=2)$. (B) Foveal OCT images of PR1.7-treated eyes $(n=2)$. DO, day of injection, predose; M1.5, $-2,-3$, month 1.5, 2, or 3 after dose; AAV, adeno-associated virus; CMV, cytomegalovirus promoter; PR1.7, promoter of 1.7 kilobases in length, based on the human red opsin gene enhancer and promoter sequences.

higher Jaws expression in cones transduced subretinally compared with cones transduced intravitreally. Temporal analysis using flicker stimulation at different frequencies showed very fast and robust photocurrent responses from $2 \mathrm{~Hz}$ up to $30 \mathrm{~Hz}$ at $8 \times 10^{16}$ photons $\mathrm{cm}^{-2} \cdot \mathrm{s}^{-1}$ (Figure $6 \mathrm{~L}$ ). In both cases, the light intensity response threshold was observed at around $1 \times 10^{15}$ photons $\mathrm{cm}^{-2} \cdot \mathrm{s}^{-1}$. While recording cones in current-clamp configuration in current zero mode enables the experimenter to record the actual resting membrane potential of the cells, we observed light-elicited hyperpolarization (Figure 6, I and J), followed by short depolarization, that was more visible with subretinally injected retinas, correlating with higher expression levels of Jaws-GFP than in intravitreally injected retinas. Jaws-induced photocurrents varied in amplitude as a function of stimulation wavelength peaking at $575 \mathrm{~nm}$, as expected (Figure $6 \mathrm{~K}$ ). Application of increasing stimulation frequencies showed that reliable photocurrents could be obtained with a return to baseline at up to $30 \mathrm{~Hz}$, at $8 \times 10^{16}$ photons $\mathrm{cm}^{-2} \cdot \mathrm{s}^{-1}$ (Figure $6 \mathrm{~L}$ ).

PR1.7 promoter drives strong and highly specific gene expression in human cones. Altogether, our data in NHPs show for the first time to our knowledge noninvasive, specific, and high-level primate foveal cone transduction compatible with optogenetic applications for vision restoration. However, as promoter activity shows important variations across species $(16,29,36)$, we deemed it necessary to validate PR1.7 in human cells and tissues. Due to the lack of a good human photoreceptor cell line or other model that could be used to test efficiency of cone promoter activity, we used 3-D retinal organoids derived from human induced pluripotent stem cells (iPSCs) (37). We generated photoreceptor-enriched retinal organoids and infected them with AAV2-7m8 vectors encoding GFP under the control of the PR1.7 promoter (Figure 7, A-C). GFP expression was observed as early as 5 days after infection and continued to increase until the experiment was terminated for analysis on day 43. GFP expression in these organoids colocalized with human CAR (hCAR) immunostaining (Figure 7, D-F). Lastly, as human retinal organoids do not represent all features of mature human retina, we validated the efficacy and specificity of the PR1.7 promoter in postmortem 

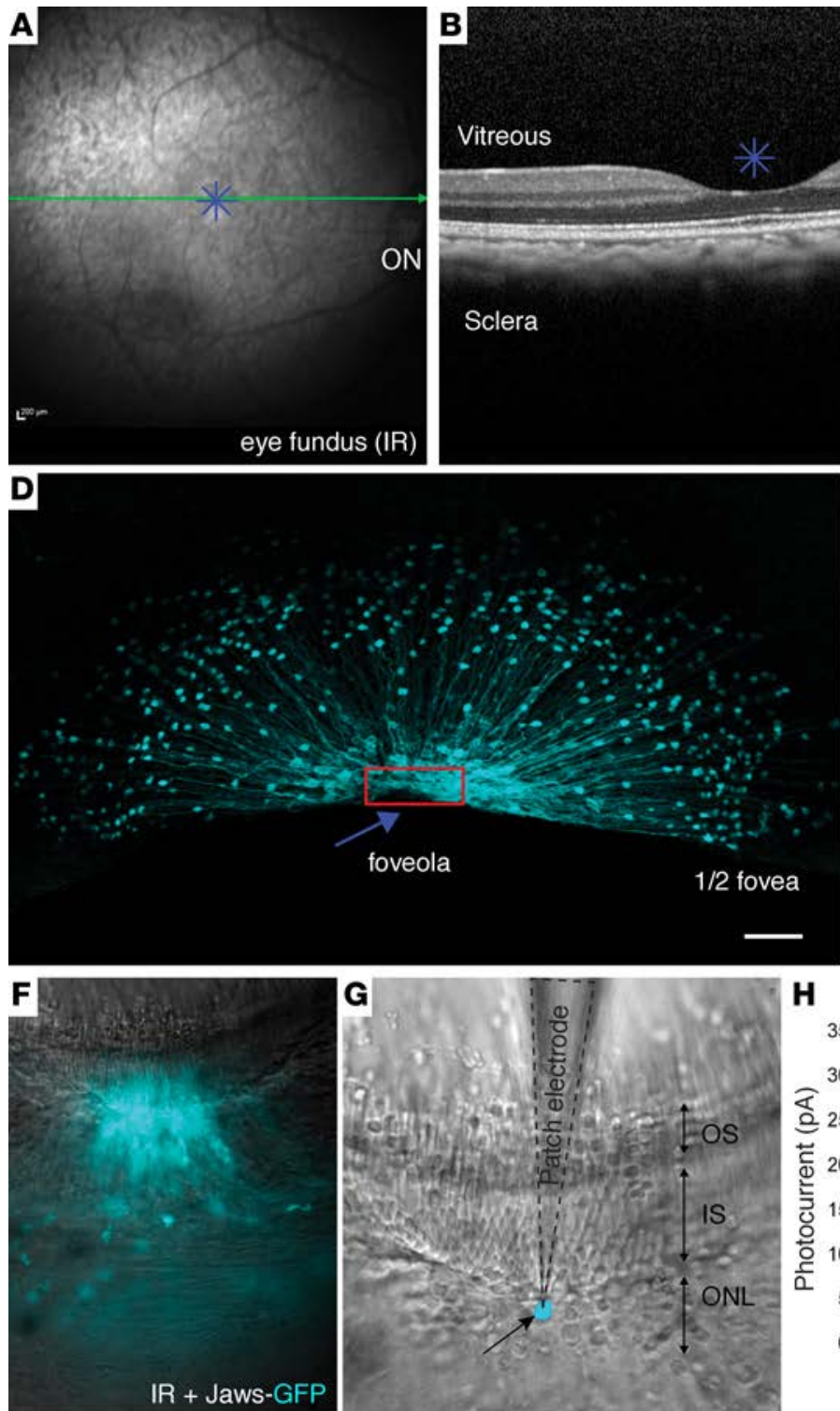

IR + Jaws-GFP
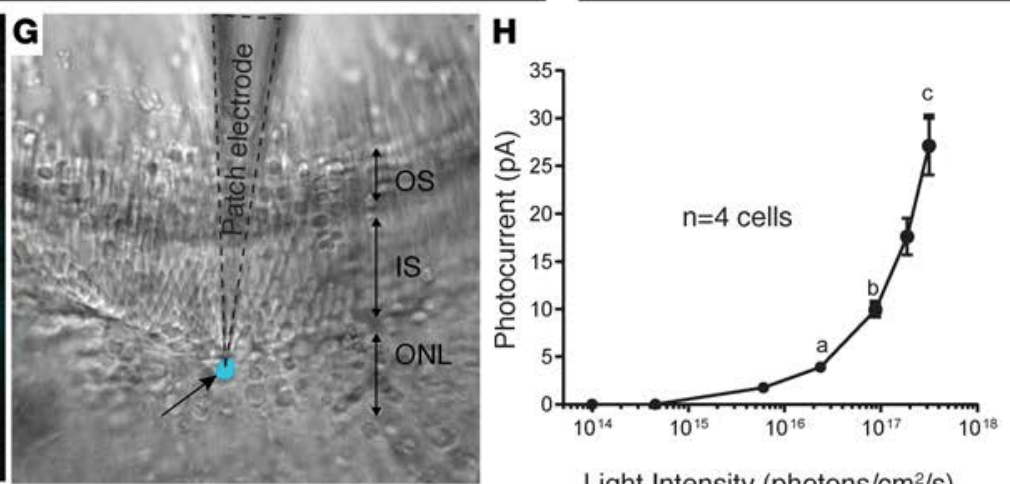

Light Intensity (photons $/ \mathrm{cm}^{2} / \mathrm{s}$ )
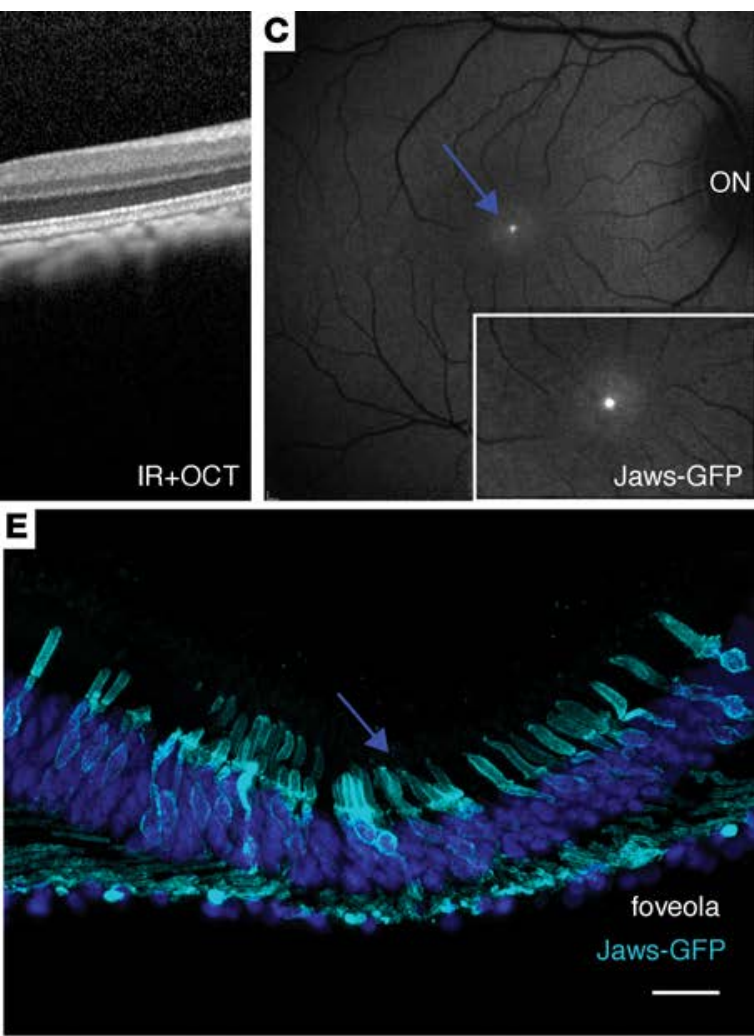

I

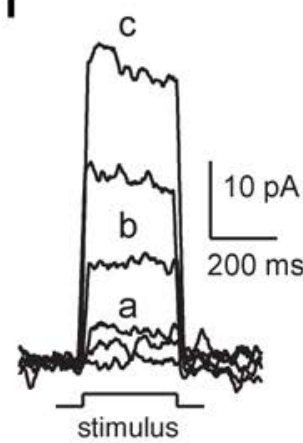

stimulus

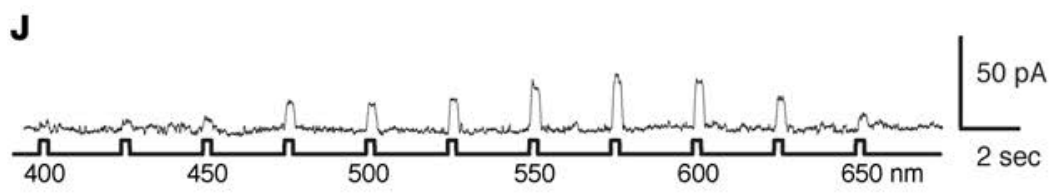

$\mathbf{K}$

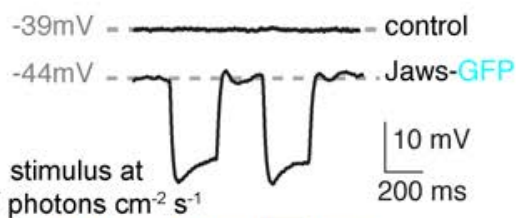

$10^{17}$ photons $\mathrm{cm}^{-2} \mathrm{~s}^{-1}$

$590 \mathrm{~nm} \longrightarrow$

Figure 5. Optogenetic activation of foveal cones using AAV2-7m8-PR1.7-Jaws-GFP. (A) Infrared eye fundus image and (B) optical coherence tomography (OCT) image of the eye injected intravitreally with AAV2-7m8-PR1.7-Jaws-GFP ( $n=1,1 \times 10^{11} \mathrm{vg}$ and $\left.n=1,1 \times 10^{10} \mathrm{vg}\right)$. (C) Eye fundus fluorescence image 2 months after injection shows Jaws-GFP expression in the fovea. Inset magnification (B and C): $\times 1.5$. (D) Half foveal flatmount showing efficient and specific foveal transduction using AAV2-7m8-PR1.7-Jaws-GFP. Scale bar: $50 \mu \mathrm{m}$. Arrow, foveola; red rectangle, close-up to the foveola shown in retinal sections in E; scale bar: $20 \mu \mathrm{m}$. (F-K) Characteristics of the cone photoreceptor light responses triggered by optogenetic stimulation of Jaws in living macaque retinas $(n=4$ cells). (F) Superimposed infrared and epifluorescence images showing strong Jaws-GFP fluorescence in the foveal cones of patched explants. (G) Infrared image of the same tissue. Patch electrode (black dotted line) is shown in contact with a Jaws-GFP' cone cell body highlighted in cyan. ONL, outer nuclear layer; IS, inner segments; OS, outer segments. (H and I) Whole-cell patch clamp recordings of Jaws-GFP-expressing macaque cones. Jaws-induced photocurrents as a function of light intensity. Orange light stimulation ranged from $1 \times 10^{14}$ to $3 \times 10^{17}$ photons $\mathrm{cm}^{-2} \cdot \mathrm{s}^{-1}$. (J) Jaws-induced photocurrents as a function of stimulation wavelength in intravitreally injected macaque eye. Stimuli were applied from $400-650 \mathrm{~nm}$, separated by $25-\mathrm{nm}$ steps, at an intensity equal to $8 \times 10^{16}$ photons $\mathrm{cm}^{-2} \cdot \mathrm{s}^{-1}$. Maximal responses were obtained at $575 \mathrm{~nm}$. (K) Jaws-CFP-expressing cones recorded in current-clamp configuration in current zero mode (with their resting membrane potential indicated in gray), displaying light-elicited hyperpolarizations followed by short depolarizations. AAV, adeno-associated virus; PR1.7, promoter of 1.7 kilobases in length, based on the human red opsin gene enhancer and promoter sequences. 
human retinal explants. Human retinal explants were cultured as described previously (38) and infected with a single drop of $1 \times 10^{10}$ particles of AAV2-7m8-PR1.7-GFP (Figure 7). Fifteen days after infection, GFP expression was analyzed on cryosections. The expression was restricted to the ONL (Figure 7I) and colocalized with M/L-opsin, a cone cell marker (Figure 7J). These data collectively point toward high efficiency and specificity of PR1.7 in leading to restricted gene expression in human cones.

\section{Discussion}

The fovea accounts for less than $1 \%$ of the retinal surface area in primates, yet it provides the input to about $50 \%$ of the cells in the primary visual cortex (1). The high concentration of cones in the fovea, the thinnest and most delicate part of the retina, allows for high-acuity vision, and it is of utmost importance to preserve the unique functions (39) and architecture (40) of the cones in this area during therapeutic interventions. Foveal cones can be targeted via different administration routes, using either subretinal or intravitreal injections $(12,16)$, but detaching the fovea might lead to mechanical damage, especially in the degenerating retina (12). For all of these reasons, ways to deliver therapeutics to the fovea, without detaching this region, are needed. Intravitreal injections are surgically simple ways to deliver therapeutics without retinal detachment. Gene therapy vectors can target the outer retina via intravitreal injections in rodents without damage to the photoreceptors $(16,17)$. However, safe and efficient gene delivery to primate cones via intravitreal injection had not been achieved so far, likely due to the substantial dilution of the vector in the vitreous and resulting loss of efficacy. The use of cell typespecific promoters that provide high-level gene expression with a lower local concentration is critical to overcome this challenge $(29,41)$.

In this study, we sought to first achieve strong and exclusive transduction of cones via noninvasive, intravitreal injection using various promoters in combination with AAV2-7m8 capsid. We selected 3 previously described promoters in view of their utility in driving gene expression in cones $(4,18,19,42$, 43) and tested them for specificity and strength of cone transduction side by side. All promoters tested in vivo in mouse retinas led to transgene expression in the photoreceptor layer when delivered subretinally. The mCAR promoter led to expression in rods and cones. Surprisingly, after intravitreal delivery, only PR1.7 maintained its specificity toward cones, while PR2.1 and mCAR gave rise to nonspecific gene expression in inner retinal neurons. $\mathrm{mCAR}$ and PR2.1 gave rise to nonspecific expression in inner retinal cells, making them unsuitable for optogenetic applications where any expression in downstream neurons would cancel out the response from the photoreceptors. Subsequent in silico analysis of TF binding sites within each promoter sequence proposed a basis for more specific transduction with PR1.7 and the observed lack of specificity with the mCAR promoter. Next, to study the ability of AAV2-7m8 equipped with the PR1.7 promoter to transduce foveal cones, we conducted gene delivery studies in macaque eyes. Complete restriction of gene expression to primate cones was achieved using AAV2-7m8-PR1.7 in the fovea via intravitreal administration.

One shortcoming with the intravitreal injection route is the higher susceptibility of AAVs administered into this compartment to interactions with the immune system compared with subretinal administration (33). It has been shown that antibody neutralization poses a barrier to intravitreal AAV vector-mediated gene delivery in NHPs, and this will likely pose a challenge for human application. We thus aimed to develop another gene delivery approach for patients who have neutralizing antibodies toward AAV2. To this aim, we tested gene delivery to foveal cones by subretinal administration of AAV9-7m8 at a distal site (Figure 8 and Table 2). We demonstrated that robust light responses could be obtained with this new delivery approach, thanks to the vector's ability to diffuse laterally and mediate expression outside of the bleb. Using the same optogenetic cone reactivation strategy, we showed that this approach also affords robust light responses mediated by Jaws but in a higher percentage of cones compared with a intravitreal route. AAV9-7m8's behavior is similar to a previously described AAV2-derived mutant that exhibits enhanced lateral spread after subretinal injections in mouse retina (44). However, in the macaque retina, we believe that the transduction beyond the bleb with AAV9-7m8 is correlated with its increased infectivity compared with its parental serotype (17) rather than with altered binding to its primary receptor.

Our in vivo findings collectively point to 3 important considerations in retinal gene delivery. First, enhanced AAV vectors, whether obtained via directed evolution (AAV2-7m8; ref. 16) or rational design (AAV9-7m8; ref. 17), can achieve therapeutic objectives where parental serotypes fail to provide sufficient gene delivery. Indeed, AAV2 and AAV9 cannot perform efficient noninvasive foveal targeting 

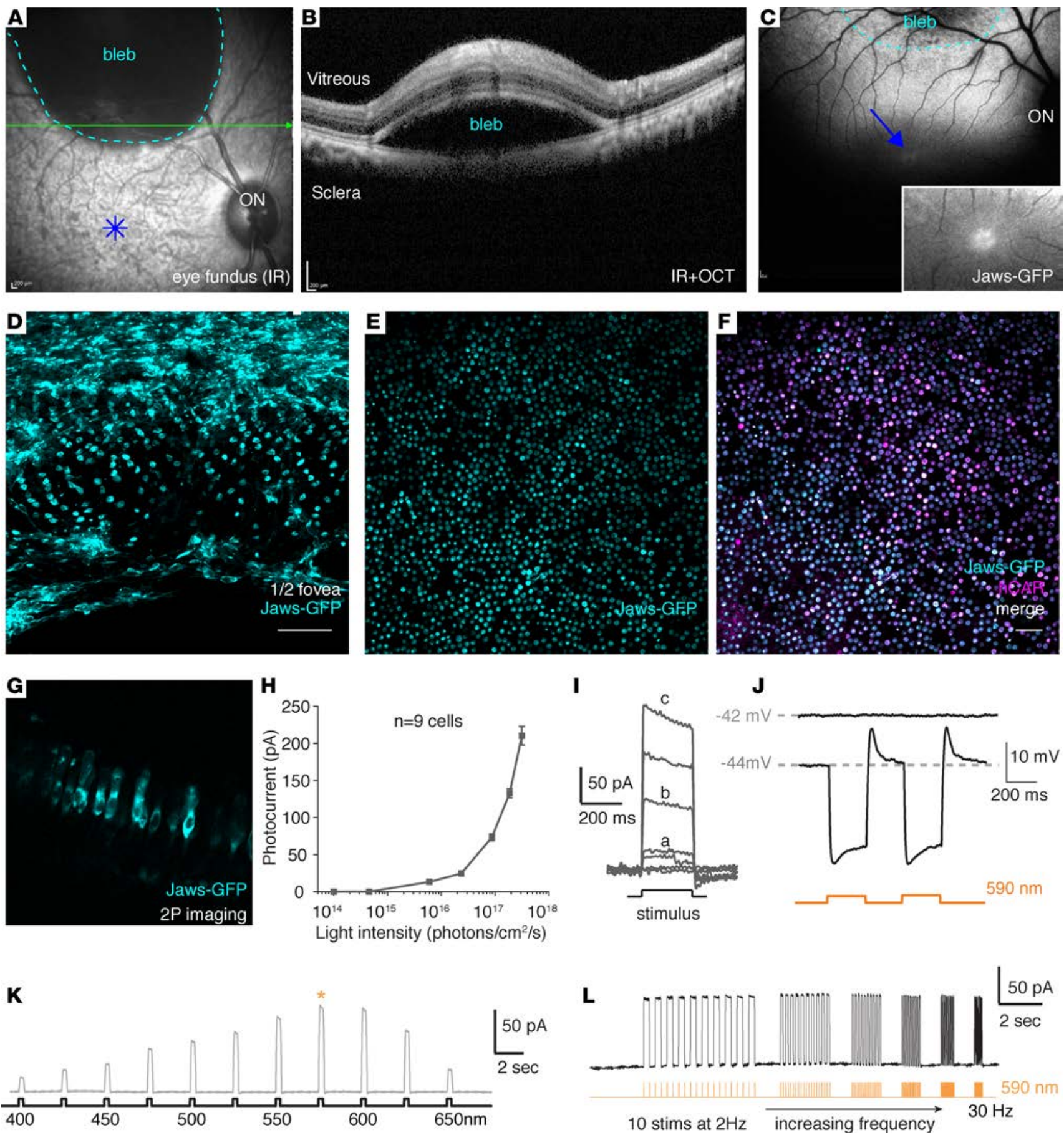

Figure 6. AAV9-7m8 transduces the fovea via delivery in a distal bleb and provides robust optogenetic light responses with PR1.7-Jaws. (A) Eye fundus infrared image and (B) optical coherence tomography (OCT) image immediately after subretinal delivery of AAV9-7m8 in peripheral retina. (C) Eye fundus fluorescence image 1 month after injection shows strong Jaws-GFP expression within the subretinal bleb and away from the injection site, including the fovea. Inset magnification: $\times 1.5$. (D-F) Foveal flatmount shows highly efficient and specific foveal transduction using subretinal AAV97m8-PR1.7-Jaws-GFP. Scale bar: $50 \mu \mathrm{m}$. (G-L) Characteristics of the light responses triggered by optogenetic stimulation of Jaws. (G) Lateral view of Jaws-expressing cones in living tissue using 2-photon imaging. ( $\mathbf{H}$ and $\mathbf{I})$ Whole-cell patch clamp recordings of Jaws-GFP ${ }^{+}$macaque cones. Jaws-induced photocurrents as a function of light intensity. Stimuli were applied from $1 \times 10^{14}$ to $3 \times 10^{17}$ photons $\mathrm{cm}^{-2} \mathrm{~s}^{-1}(n=9$ cells from 2 retinas of 2 animals). (J) Jaws-GFP+ cones recorded in current-clamp configuration in current zero mode (with resting membrane potential indicated in gray), displaying light-elicited hyperpolarizations followed by short depolarizations. (K) Jaws-induced photocurrents as a function of stimulation wavelength in subretinally injected macaque eye. Stimuli were applied from $400-650 \mathrm{~nm}$, separated by $25-\mathrm{nm}$ steps, at an intensity equal to $8 \times 10^{16}$ photons $\mathrm{cm}^{-2} \cdot \mathrm{s}^{-1}$. Maximal responses were obtained at $575 \mathrm{~nm}$ (asterisk). (L) Characterization of temporal properties. Modulation of Jaws-induced membrane photocurrents at increasing stimulation frequencies in Jaws-expressing macaque cones, from $2-30 \mathrm{~Hz}$, at $8 \times 10^{16}$ photons $\mathrm{cm}^{-2}$. $\mathrm{s}^{-1}$. AAV, adeno-associated virus; PR1.7, promoter of 1.7 kilobases in length, based on the human red opsin gene enhancer and promoter sequences. IR, infrared. 

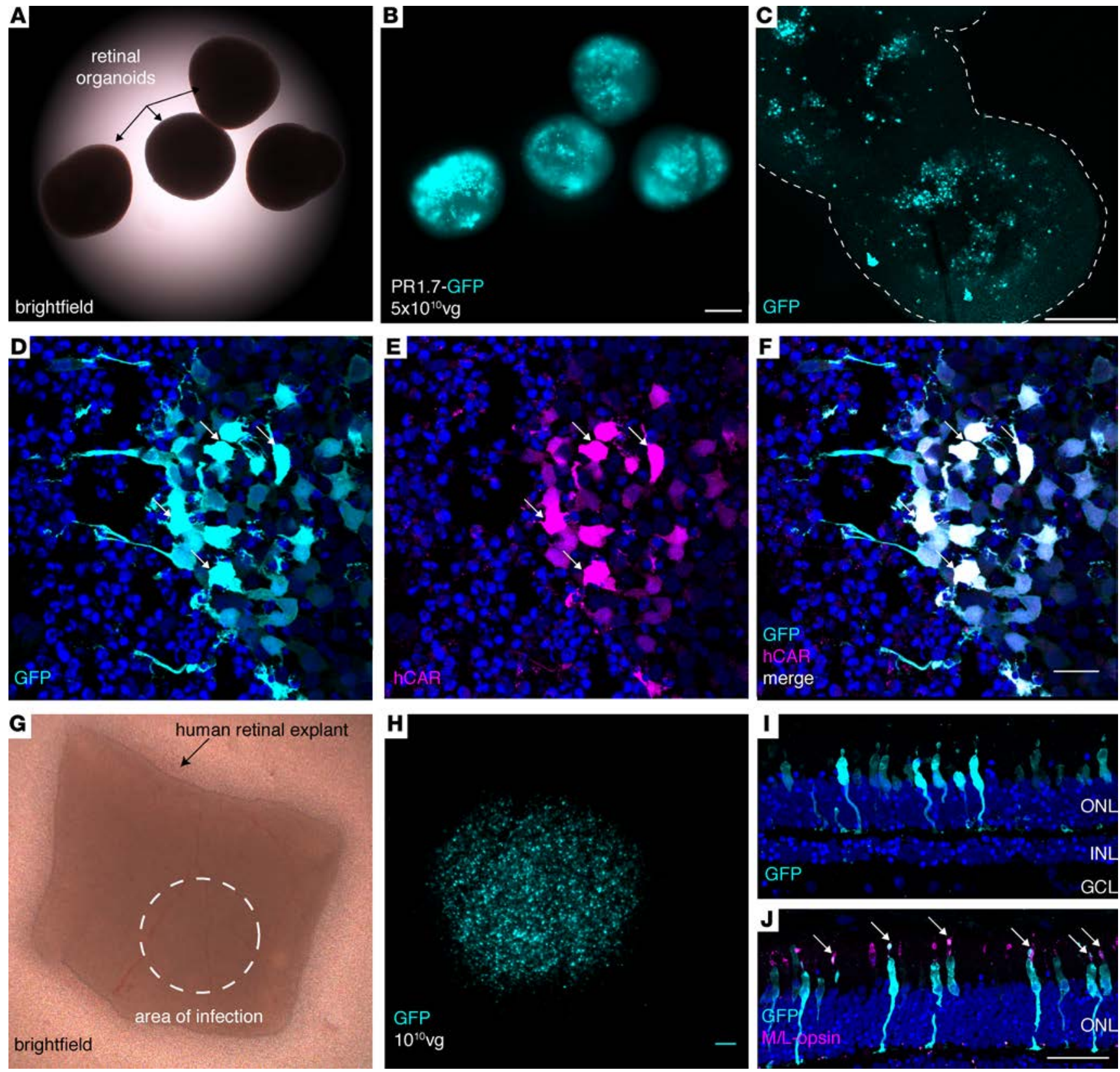

Figure 7. Performance of AAV2-7m8-PR1.7 vector-promoter combination in human cones. (A-C) GFP expression in human induced pluripotent stem cell-derived (iPSC-derived) retinal organoids ( $n=10$ organoids) infected with AAV2-7m8-PR1.7-GFP. (A) Brighfield, (B) epifluorescence, and (C) confocal images of 43-day-old whole mount organoids infected with AAV2.7m8-PR1.7-GFP at day 28 with a dose of $5 \times 10^{10} \mathrm{vg} / \mathrm{organoid}$. Scale bar: $200 \mu \mathrm{m}$ in $\mathbf{A}$ and $\mathbf{B}$, and $250 \mu \mathrm{m}$ in $\mathbf{C}$. Outline in $\mathbf{C}$ represents the edges of the organoids (D-F) Retinal organoid cryosections for visualization of GFP expression (cyan). Transduced cones are visualized by superimposition of GFP (cyan) and human cone arrestin (hCAR) immunostaining (magenta). Scale bar: 20 $\mu \mathrm{m}$ in D-F. Arrows represent colocalization of GFP and hCAR stainings. (G-I) Efficient and specific transduction of human cones in postmortem retinal explants. (G) Postmortem human retinal explant placed in culture. Dashed circle shows the approximate area where $1 \times 10^{10}$ viral particles were deposited onto the explant ( $n=2$ explants from 2 eyes of a single donor). (H) Close-up of the transduced area showing high-level GFP fluorescence in region of the explant in contact with the vector. Scale bar: $100 \mu \mathrm{m}$. (I) GFP expression (cyan) is restricted to the photoreceptor layer as shown by DAPI (blue) staining. (J) GFP is expressed in cones as shown by colocalization of GFP staining of cone markers, namely M/L opsin. Scale bar: $50 \mu \mathrm{m}$ in I-J. Arrows represent colocalization of GFP and M/L opsin stainings. AAV, adeno-associated virus; vg, viral genome; PR1.7, promoter of 1.7 kilobases in length, based on the human red opsin gene enhancer and promoter sequences.

$(30,31)$ while $7 \mathrm{~m} 8$ modified vectors bridge this gap. Second, strong cell type-specific promoters allow dose sparing (Table 3) important for the safety of gene therapy (i.e., avoiding immune response). Third, our study shows the nonnegligible impact of the vector administration route on transgene expression patterns. Finally, to complement our in vivo results in animals, we performed a battery of ex vivo tests 


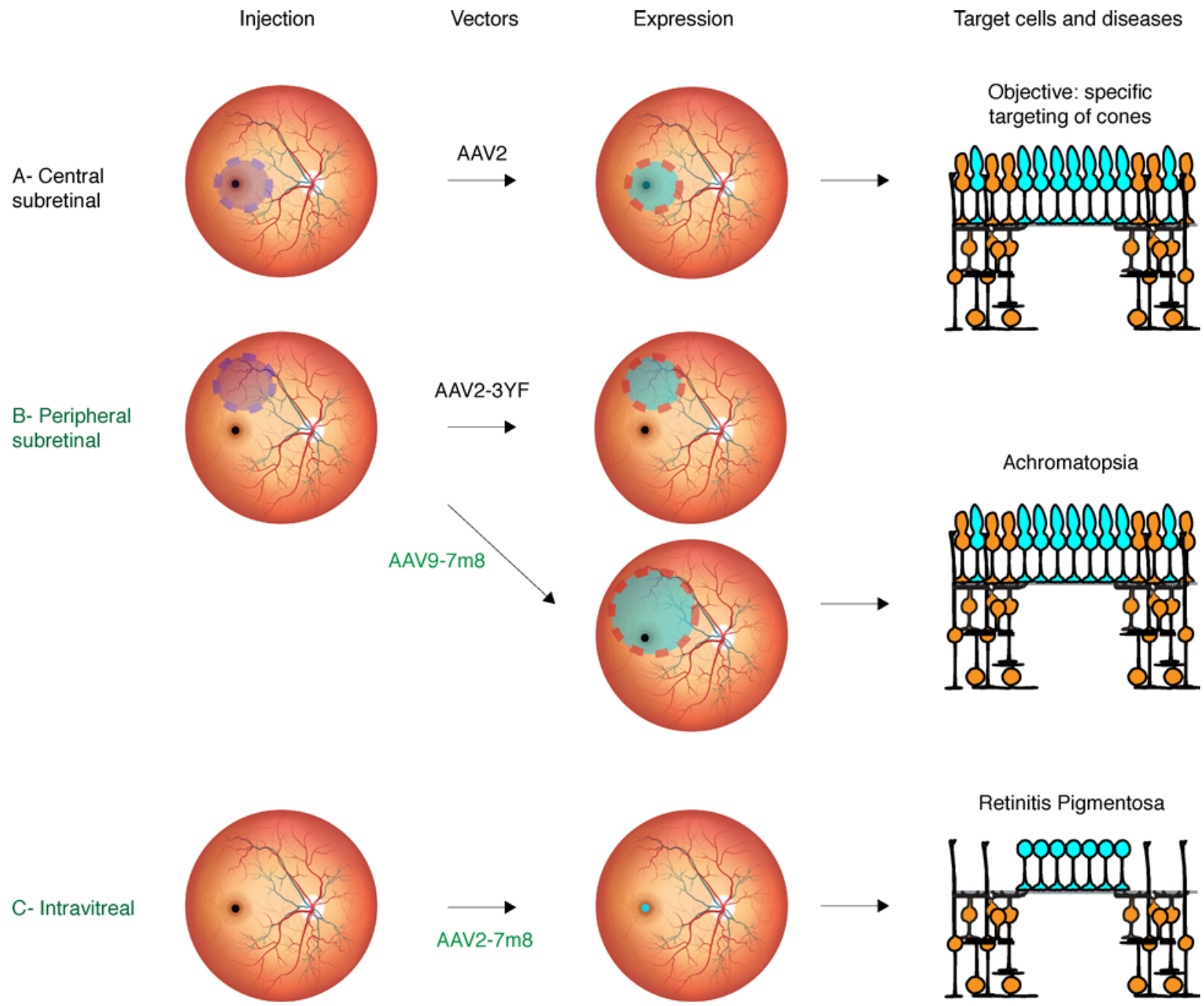

Figure 8. Vector delivery strategies to meet therapeutic gene expression requirements. (A) Central subretinal injection is the most risky and can be associated to adverse effects in the macula. (B) Peripheral subretinal injection using classical vectors does not reach the fovea; however, use of AAV9-7m8 is a promising strategy for achromatopsia patients. (C) Intravitreal injection is surgically simpler and the safest administration route to transduce cones of the foveola, the region responsible for high-acuity vision. It is a preferred delivery approach for retinitis pigmentosa patients to benefit from optogenetic therapy. AAV, adeno-associated virus.

in human tissues that, in combination with in vivo experiments, constitute a versatile platform for validating gene therapy for clinical application. The vector-promoter combinations described here will find utility in all retinal diseases where cone targeting is desired. Each administration route and vector can be considered based on the serological state of the patient and natural history of the targeted disease (Table 2). The combination of PR1.7 and AAV2-7m8 is ideal for therapeutic gene expression in human foveal cones when delivered into the vitreous (Figure 8) and can be an ideal way to reanimate remaining dormant cones with optogenetics in retinitis pigmentosa (4). Since cones subsist in both the center and the periphery in achromatopsia, gene delivery in the periphery using AAV9-7m8-PR1.7 can be more efficacious, as it would deliver the therapeutic gene into both the foveal and peripheral cones (Figure 8).

\section{Methods}

$A A V$ production. AAV vectors were produced as previously described using the cotransfection method and purified by iodixanol gradient ultracentrifugation (45). AAV vector stocks were titered by quantitative PCR (qPCR) (46) using SYBR Green (Thermo Fischer Scientific).

Animals and intraocular injections. WT C57BL6/j mice (Janvier Labs) or rd10 mice (bred and raised in the animal facility of the Vision Institute) were used for this study. For eye injections ( $n=6$ eyes/condition), 
Table 2. Adeno-associated viral (AAV) vector administration strategies for cone-directed gene therapy in primates

\begin{tabular}{|c|c|c|c|c|}
\hline Injection route & Peripheral subretinal & Central subretinal & Peripheral subretinal (near macula) & Intravitreal \\
\hline $\begin{array}{l}\text { Therapeutic gene } \\
\text { expression }\end{array}$ & Peripheral & Central (macula-fovea) & Peripheral and central & Central \\
\hline Potential capsids & $\begin{array}{l}\text { AAV2-3YF }(18,43) \\
\text { AAV9 }(18,30,43)\end{array}$ & AAV2 in clinical trials (7-9) & AAV9-7m8 as used in this study & $\begin{array}{l}\text { AAV2 and its tyrosine mutants } \\
\qquad(7,32) \\
\text { AAV2-7m8 as used in this study }\end{array}$ \\
\hline Advantages & $\begin{array}{c}\text { Immune privilege } \\
\text { High-level therapeutic gene } \\
\text { expression }\end{array}$ & $\begin{array}{c}\text { Immune privilege } \\
\text { High-level therapeutic gene } \\
\text { expression } \\
\text { Foveal transduction } \\
\text { High-acuity vision }\end{array}$ & $\begin{array}{l}\text { Immune privilege } \\
\text { High-level therapeutic gene } \\
\text { expression } \\
\text { Larger expression area that } \\
\text { includes the fovea } \\
\text { High-acuity vision } \\
\text { Not invasive to the fovea }\end{array}$ & $\begin{array}{c}\text { Noninvasive } \\
\text { Potential high-acuity vision } \\
\text { Controlled area of expression } \\
\text { pattern }\end{array}$ \\
\hline Disadvantages & $\begin{array}{l}\text { Invasive } \\
\text { No foveal transduction } \\
\text { Low-acuity vision }\end{array}$ & $\begin{array}{l}\text { Invasive, risk of adverse } \\
\text { effects such as macular } \\
\text { thinning (11) }\end{array}$ & & $\begin{array}{l}\text { Presence of NAbs in the } \\
\text { vitreous (use of glucocorticoids } \\
\text { could prevent antivector } \\
\text { immune response if patient is } \\
\text { seropositive for AAV2; ref. 50) } \\
\text { Lower gene expression than } \\
\text { subretinal }\end{array}$ \\
\hline $\begin{array}{l}\text { Potential target } \\
\text { diseases and } \\
\text { applications }\end{array}$ & \multicolumn{4}{|c|}{$\begin{array}{l}\text { Retinitis pigmentosa: optogenetic vision restoration (Jaws) }(4,15) \\
\text { Achromatopsia: CNGA3 or CNGB3 }(43)\end{array}$} \\
\hline
\end{tabular}

NAbs, neutralizing antibodies; CNGA3, cyclic nucleotide gated channel $\alpha$ 3; CNGB3: cyclic nucleotide gated channel $\beta 3$.

6-week-old female mice were anesthetized by isofluorane inhalation. Pupils were dilated, and a 33-gauge needle was inserted into the eye to deliver $2 \mu 1$ of AAV vector solution intravitreally or $1 \mu 1$ subretinally.

Cynomolgus macaques (Noveprim, Mauritius) were first selected based on the absence of neutralizing antibody titers against AAV. Prior to surgery, , they were anesthetized with an intramuscular injection of Ketamine, $10 \mathrm{mg} / \mathrm{kg}$ (Imalgene 1000, Merial) and Xylazine $0.5 \mathrm{mg} / \mathrm{kg}$ (Rompun $2 \%$, Bayer). Anesthesia was maintained with an intravenous injection of propofol, $1 \mathrm{ml} / \mathrm{kg} / \mathrm{h}$ (PropoVet Multidose $10 \mathrm{mg} / \mathrm{ml}$, Zoetis). Then, their pupils were dilated and their eyelids were kept open using eyelid speculum. A 1-ml syringe equipped with a 32-mm, 27-gauge needle was used for intravitreal injections. The needle was inserted into the sclera approximately $2 \mathrm{~mm}$ posterior to the limbus to deliver $100 \mu \mathrm{l}$ of the viral vector solution. Finally, the needle was slowly removed. Animals did not receive local corticosteroid injections.

For subretinal AAV injections, two 25-gauge vitrectomy ports were set approximately $2 \mathrm{~mm}$ posterior to the limbus, one for the endo-illumination probe and the other for the subretinal cannula. A 1-ml Hamilton syringe equipped with a 25-gauge subretinal cannula with a 41-gauge tip was used for the injection. The endoillumination probe and cannula were introduced into the eye. The viral vector solution $(50 \mu l)$ was

Table 3. Summary of studies involving intravitreal injections with the objective of targeting photoreceptors in primates

\begin{tabular}{|c|c|c|c|}
\hline AAV and expression cassette & Dose (vg/eye) & Results & References \\
\hline AAV2-7m8-CMV-GFP & $5 \times 10^{12} \mathrm{vg}$ & Transduction of photoreceptors & 16 \\
\hline AAV2-7m8-CMV/CBA-GFP & $1 \times 10^{12} \mathrm{vg}$ & Transduction of photoreceptors & 29 \\
\hline AAV2-CBA-GFP & $4.5 \times 10^{10} \mathrm{vg}$ & $\begin{array}{l}\text { Transduction of photoreceptors in the injected area. } \\
\text { AAV injections were under the ILM (referred to as sublLM injections). }\end{array}$ & 32 \\
\hline AAV2-3YF-CBA-GFP & $9.5 \times 10^{11} \mathrm{vg}$ & $\begin{array}{l}\text { Transduction of PRs in the ILM peeled area, following vitrectomy and } \\
\text { surgical ILM peeling. AAV injections were done } 1 \text { month after surgery. }\end{array}$ & 51 \\
\hline
\end{tabular}


injected subretinally to create a bleb either below or above the fovea. The instruments were then withdrawn. Eyes received corticosteroid treatment (47) that consisted of a laterobulbar injection of $12 \mathrm{mg}$ of Kenacort (Bristol-Myers Squibb) except right eye of NHP5.

After subretinal or intravitreal vector administration, opthtalmic steroid and antibiotic ointments (Fradexam, TVM) were applied to the corneas after injections.

In vivo macaque eye imaging. After pupil dilation, a Spectralis HRA+OCT system (Heidelberg Engineering) was used to acquire OCT images and fluorescent images of GFP using the Fundus Autofluoresence mode (excitation wavelength of $488 \mathrm{~nm}$ and barrier filter of $500 \mathrm{~nm}$ ).

Two-photon imaging and ex vivo electrophysiological recordings of macaque retinas. A 2-photon microscope equipped with a $40 \times$ water immersion objective (LUMPLFLN40 $/ \mathrm{W} / 0.80$, Olympus) with a pulsed femto-second laser (InSight DeepSee, Newport Corporation) was used for imaging $\mathrm{GFP}^{+}$retinal cells from whole-mount retinas (with photoreceptor cell side up) or retina slices (vertical sections). AAV-treated macaque retinas were isolated and later imaged in oxygenized $\left(95 \% \mathrm{O}_{2}, 5 \% \mathrm{CO}_{2}\right)$ Ames medium (MilliporeSigma). For live 2-photon imaging, retinas were placed in the recording chamber of the microscope, and Z-stacks were acquired using the excitation laser at a wavelength of $930 \mathrm{~nm}$. Images were processed offline using ImageJ (NIH). For whole-cell patch-clamp recordings, an Axon Multiclamp 700B amplifier was used. Electrodes were made from borosilicate glass (BF100-50-10, Sutter Instruments) and pulled to 6-9 M . Pipettes were filled with $115 \mathrm{mM} \mathrm{K}$ gluconate, $10 \mathrm{mM} \mathrm{KCl}, 1 \mathrm{mM} \mathrm{MgCl} 2,0.5 \mathrm{mM} \mathrm{CaCl} 2,1.5$ $\mathrm{mM}$ EGTA, $10 \mathrm{mM}$ HEPES, and $4 \mathrm{mM}$ ATP-Na2 (pH 7.2). Cells were clamped at a potential of -40 $\mathrm{mV}$ in voltage-clamp configuration or recorded in current-clamp (current 0 ) configuration. Retinas were dark-adapted at least 30 minutes in the recording chamber prior to recordings.

Human iPSC cultures. We have generated retinal organoids from human iPSCs based on a previously published protocol (37). Clone hiPSC-2 was expanded and differentiated on fibroblast feeders from postnatal human foreskins (ATCC CRL 2429) in proneural medium, as already described (37). Starting from highly confluent adherent iPSC cultures and in the absence of fibroblast growth factor 2 (FGF2), self-forming retinal organoids can be identified after 2 weeks. At this point, the organoids were mechanically isolated and cultured in 3-D conditions for up to 43 days. FGF2 was supplemented to the medium in 3 conditions for 7 days after the mechanical isolation of the organoids to promote their growth. The retinal organoids were infected at day 28 of differentiation at a dose of $5 \times 10^{10} \mathrm{vg} /$ organoid with AAV2-7m8 vectors carrying the GFP gene under the control of the PR1.7 promoter. DAPT (10 $\mu \mathrm{M}$; Selleck) was added to the medium for a week from day 28 on to promote cell cycle arrest of the existent cell populations. Fluorescence intensity was observed for the first time 5 days after infection and continued to increase up to day 43

Human postmortem retinal explants. Human retinal explants were prepared using a previously described protocol (38). Briefly, eyes were dissected in $\mathrm{CO}_{2}$ independent medium (Thermo Fischer Scientific). The anterior parts were removed, and retina was isolated and cut into small pieces. These explants were placed photoreceptor side-up on a Transwell cell culture insert (Corning), and $2 \mathrm{ml}$ of neurobasal medium (Thermo Fischer Scientific) supplemented with B27 (Thermo Fischer Scientific) were added to each well below each explant. The following day, each explant was infected with a single 0.5- $\mu 1$ drop of AAV2-7m8-PR1.7GFP containing $1 \times 10^{10}$ viral particles. Vector-infected explants were incubated for 10-15 days to allow GFP expression, which was checked using an epifluorescence macroscope.

Histology, IHC, and microscopy. Mouse eyes were enucleated and immediately fixed in 10\% formalin and $4 \%$ formaldehyde (Sigma) for 2 hours for cryosections. Macaque retinas were fixed after dissection in $4 \%$ formaldehyde (Sigma) for 3 hours. Retinal organoids and human retinal explants were rinsed in PBS at the end of their culture periods and fixed in $4 \%$ paraformaldehyde for 10 minutes. For cryosections, mouse and macaque retinas, retinal organoids, and human retinal explants were immersed in PBS-30\% sucrose (Sigma) overnight at $4^{\circ} \mathrm{C}$. Mouse eye cups, human retinal explants, and macaque retinas were embedded optimal cutting temperature compound (Microm Microtech France) compound and frozen in liquid nitrogen, while retinal organoids were embedded in 7.5\% gelatin (Sigma) and 10\% sucrose (Sigma) in PBS and frozen in dry ice-cold isopentane (Merck Millipore). Vertical sections (10 $\mu \mathrm{m}$-thick) were cut with a Microm cryostat. After incubation in the blocking buffer, sections were incubated with primary antibodies overnight at $4^{\circ} \mathrm{C}$ : hCAR antibody (gift from Cheryl Craft, University of Southern California, Los Angeles, USA), M/L opsin antibody (Merck Millipore, AB5405), and mouse cone arrestin antibody (Merck Millipore, AB15282). After multiple washes of the sections, the secondary antibody Alexa Fluor 594 (A10040, Thermo Fischer Scientif- 
ic) and DAPI were added, followed by several washes. Retinal flatmounts or cryosections were mounted in Vectashield mounting medium (Vector Laboratories) for fluorescence microscopy, and retinal sections were visualized using an Olympus Upright confocal microscope and then analyzed with Fiji software. Three-dimensional projections of the fovea were created with Imaris software (Bitplane).

In silico identification of potential regulatory elements and transcriptomic analysis. TF binding site analysis was performed on red opsin gene promoter sequence - PR2.1 and PR1.7 sequences - and the cone arrestin 3 genomic region. The TRANSFAC database 8.3 (http://alggen.1si.upc.es/) was used for TF binding site prediction. Each TF from the predicted list was analyzed using the Knowledge Base for Sensory System (KBaSS, http://kbass.institut-vision.org/KBaSS/transcriptomics/index.php) to select those expressed in human retina using the transcriptomic experiment RNG209 (48). A filter was used to retain TFs with a signal intensity value superior to 40 units in the sample prepared from the experiment RNG209 after normalization by robust multi-array average (RMA) as previously described (49). In this experiment, human retinal specimens used as controls were postmortem specimens collected within 12 hours following death of patients with no past medical history of eye disease or diabetes. Nineteen samples were collected from 19 eyes, representing 17 patients. Sex ratio was 12 men/ 7 women with a mean age of 61 years (range $25-78$ years).

Statistics. Data were analyzed using ANOVA test in Graphpad Prism (multiple comparison, Tukey correction). Error bars on the graphs show the \pm SEM. $P<0.033$ was considered significant.

Study approval. For animals, the experiments were realized in accordance with the NIH Guide for Care and Use of Laboratory Animals (National Academies Press, 2011). The protocols were approved by the Local Animal Ethics Committees and conducted in accordance with Directive 2010/63/EU of the European Parliament.

Postmortem human ocular globes from donors were acquired from the School of Surgery (Ecole de Chirugie, Assitance Publique Hôpitaux de Paris, Paris, France). The protocol was approved by the IRBs of the School of Surgery and the Quinze-Vingts National Ophtalmology Hospital (Paris, France). All experiments on postmortem human retinal explants were performed according to the local regulations, as well as the guidelines of the Declaration of Helsinki.

\section{Author contributions}

$\mathrm{HK}, \mathrm{MGH}$, and DD designed experiments. $\mathrm{HK}, \mathrm{CW}$, and $\mathrm{MD}$ generated plasmid constructs used in the study. $\mathrm{HK}$ performed all surgical procedures on mice. MD optimized neutralizing antibody detection protocol. SB performed surgical procedures on macaques. $\mathrm{CJ}$ and EB performed macaque eye imaging and ophthalmic examinations. MGH prepared human iPSC-derived retinal organoids and optimized their AAV-mediated infection and characterization. AC performed patch-clamp recordings in macaque retinal explants. SR analyzed promoter sequences and provided lists of TF binding sites. HK, SR, and MGH analyzed these data. HK and VF prepared postmortem macaque and human retinal explants. HK performed histology and imaging on mouse, macaque, and human tissue. HK and DD designed the study and wrote the manuscript. HK prepared the figures and tables. OG, SP, JD, and JAS provided scientific input and gave feedback on the manuscript.

\section{Acknowledgments}

This study was supported by The Foundation Fighting Blindness (Wynn-Gund translational research award), AFM-Téléthon (Young Researcher PhD Fellowship to HK), Marie Curie CIG (334130, RETINAL GENE THERAPY), INSERM, Labex-Lifesenses (ANR-10-LABX-65), the Agence Nationale pour la Recherche - Recherche Hospitalo-Universitaire en santé (RHU) (Light4Deaf), Fondation NRJ and ERC StGs (OPTOGENRET and REGENETHER). We thank Emilie Kéomani and Camille Robert for assistance with the production of AAV vectors, Amélie Slembrouck and Laure Guibbal for assistance on hiPSC maintenance, Estelle Dias and Marie-Laure Niepon (Histology Platform) for assistance on retinal cryosections. We also thank Claire-Maëlle Fovet and Joanna Demilly for technical assistance on the MIRCEN NHP platform, Stéphane Fouquet for technical assistance on the Imaging Platform of the Vision Institute and Laure Pacot for help with cell counting on confocal images. We are thankful to Thierry Léveillard for access to the human retinal transcriptomics data in the KBaSS.

Address correspondence to: Deniz Dalkara, Vision Institute, 17 rue Moreau, Paris, 75012, France. Phone: 33153462532; Email: deniz.dalkara@gmail.com. 
1. Wässle H, Grünert U, Röhrenbeck J, Boycott BB. Cortical magnification factor and the ganglion cell density of the primate retina. Nature. 1989;341(6243):643-646.

2. Kolb H, Zhang L, Dekorver L, Cuenca N. A new look at calretinin-immunoreactive amacrine cell types in the monkey retina. J Comp Neurol. 2002;453(2):168-184.

3. Wikler KC, Williams RW, Rakic P. Photoreceptor mosaic: number and distribution of rods and cones in the rhesus monkey retina. J Comp Neurol. 1990;297(4):499-508.

4. Busskamp V, et al. Genetic reactivation of cone photoreceptors restores visual responses in retinitis pigmentosa. Science. 2010;329(5990):413-417.

5. Byrne LC, et al. Viral-mediated RdCVF and RdCVFL expression protects cone and rod photoreceptors in retinal degeneration. J Clin Invest. 2015;125(1):105-116.

6. Komáromy AM, et al. Gene therapy rescues cone function in congenital achromatopsia. Hum Mol Genet. 2010;19(13):2581-2593

7. Maguire AM, et al. Safety and efficacy of gene transfer for Leber's congenital amaurosis. $N$ Engl J Med. 2008;358(21):2240-2248.

8. Bainbridge JW, et al. Effect of gene therapy on visual function in Leber's congenital amaurosis. $N$ Engl J Med. 2008;358(21):2231-2239.

9. Cideciyan AV, et al. Human gene therapy for RPE65 isomerase deficiency activates the retinoid cycle of vision but with slow rod kinetics. Proc Natl Acad Sci USA. 2008;105(39):15112-15117.

10. Bainbridge JW, et al. Long-term effect of gene therapy on Leber's congenital amaurosis. N Engl J Med. 2015;372(20):1887-1897.

11. Jacobson SG, et al. Improvement and decline in vision with gene therapy in childhood blindness. $N$ Engl J Med. 2015;372(20):1920-1926.

12. Jacobson SG, et al. Gene therapy for leber congenital amaurosis caused by RPE65 mutations: safety and efficacy in 15 children and adults followed up to 3 years. Arch Ophthalmol. 2012;130(1):9-24.

13. MacLaren RE, et al. Retinal gene therapy in patients with choroideremia: initial findings from a phase $1 / 2$ clinical trial. Lancet. 2014;383(9923):1129-1137.

14. Duncan JL. Visual Consequences of Delivering Therapies to the Subretinal Space. JAMA Ophthalmol. 2017;135(3):242-243.

15. Chuong AS, et al. Noninvasive optical inhibition with a red-shifted microbial rhodopsin. Nat Neurosci. 2014;17(8):1123-1129.

16. Dalkara D, et al. In vivo-directed evolution of a new adeno-associated virus for therapeutic outer retinal gene delivery from the vitreous. Sci Transl Med. 2013;5(189):189ra76.

17. Khabou $\mathrm{H}$, et al. Insight into the mechanisms of enhanced retinal transduction by the engineered AAV2 capsid variant $-7 \mathrm{~m} 8$. Biotechnol Bioeng. 2016;113(12):2712-2724.

18. Ye GJ, et al. Cone-Specific Promoters for Gene Therapy of Achromatopsia and Other Retinal Diseases. Hum Gene Ther. 2016;27(1):72-82.

19. Ye GJ, et al. Safety and Biodistribution Evaluation in CNGB3-Deficient Mice of rAAV2tYF-PR1.7-hCNGB3, a Recombinant AAV Vector for Treatment of Achromatopsia. Hum Gene Ther Clin Dev. 2016;27(1):27-36.

20. Kolstad KD, et al. Changes in adeno-associated virus-mediated gene delivery in retinal degeneration. Hum Gene Ther. 2010;21(5):571-578.

21. Vacca O, et al. AAV-mediated gene delivery in Dp71-null mouse model with compromised barriers. Glia. 2014;62(3):468-476.

22. Satoh $\mathrm{S}$, et al. The spatial patterning of mouse cone opsin expression is regulated by bone morphogenetic protein signaling through downstream effector COUP-TF nuclear receptors. J Neurosci. 2009;29(40):12401-12411.

23. Pickrell SW, Zhu X, Wang X, Craft CM. Deciphering the contribution of known cis-elements in the mouse cone arrestin gene to its cone-specific expression. Invest Ophthalmol Vis Sci. 2004;45(11):3877-3884.

24. von Mering C, et al. STRING 7--recent developments in the integration and prediction of protein interactions. Nucleic Acids Res. 2007;35(Database issue):D358-D362.

25. Blazek E, Mittler G, Meisterernst M. The mediator of RNA polymerase II. Chromosoma. 2005;113(8):399-408.

26. Bourbon HM, et al. A unified nomenclature for protein subunits of mediator complexes linking transcriptional regulators to RNA polymerase II. Mol Cell. 2004;14(5):553-557.

27. Yuan CX, Ito M, Fondell JD, Fu ZY, Roeder RG. The TRAP220 component of a thyroid hormone receptor- associated protein (TRAP) coactivator complex interacts directly with nuclear receptors in a ligand-dependent fashion. Proc Natl Acad Sci USA. 1998;95(14):7939-7944.

28. Viets K, Eldred K, Johnston RJ. Mechanisms of Photoreceptor Patterning in Vertebrates and Invertebrates. Trends Genet. 2016;32(10):638-659.

29. Ramachandran PS, et al. Evaluation of Dose and Safety of AAV7m8 and AAV8BP2 in the Non-Human Primate Retina. Hum Gene Ther. 2017;28(2):154-167.

30. Vandenberghe LH, et al. AAV9 targets cone photoreceptors in the nonhuman primate retina. PLoS ONE. 2013;8(1):e53463.

31. Vandenberghe LH, et al. Dosage thresholds for AAV2 and AAV8 photoreceptor gene therapy in monkey. Sci Transl Med. 2011;3(88):88ra54.

32. Boye SE, et al. Highly Efficient Delivery of Adeno-Associated Viral Vectors to the Primate Retina. Hum Gene Ther. 2016;27(8):580-597.

33. Kotterman MA, Yin L, Strazzeri JM, Flannery JG, Merigan WH, Schaffer DV. Antibody neutralization poses a barrier to intravitreal adeno-associated viral vector gene delivery to non-human primates. Gene Ther. 2015;22(2):116-126.

34. Sengupta A, et al. Red-shifted channelrhodopsin stimulation restores light responses in blind mice, macaque retina, and human retina. EMBO Mol Med. 2016;8(11):1248-1264.

35. Bell CL, Gurda BL, Van Vliet K, Agbandje-McKenna M, Wilson JM. Identification of the galactose binding domain of the adeno-associated virus serotype 9 capsid. J Virol. 2012;86(13):7326-7333.

36. Yin L, et al. Intravitreal injection of AAV2 transduces macaque inner retina. Invest Ophthalmol Vis Sci. 2011;52(5):2775-2783

37. Reichman S, et al. From confluent human iPS cells to self-forming neural retina and retinal pigmented epithelium. Proc Natl Acad Sci USA. 2014;111(23):8518-8523.

38. Fradot M, et al. Gene therapy in ophthalmology: validation on cultured retinal cells and explants from postmortem human eyes. Hum Gene Ther. 2011;22(5):587-593. 
39. Sinha R, Hoon M, Baudin J, Okawa H, Wong RO, Rieke F. Cellular and Circuit Mechanisms Shaping the Perceptual Properties of the Primate Fovea. Cell. 2017;168(3):413-426.e12.

40. Anderson DH, Fisher SK. The relationship of primate foveal cones to the pigment epithelium. J Ultrastruct Res. 1979;67(1):23-32

41. Tenenbaum L, Lehtonen E, Monahan PE. Evaluation of risks related to the use of adeno-associated virus-based vectors. Curr Gene Ther. 2003;3(6):545-565.

42. Boyd RF, et al. Photoreceptor-targeted gene delivery using intravitreally administered AAV vectors in dogs. Gene Ther. 2016;23(2):223-230.

43. Ye GJ, et al. Safety and Biodistribution Evaluation in Cynomolgus Macaques of rAAV2tYF-PR1.7-hCNGB3, a Recombinant AAV Vector for Treatment of Achromatopsia. Hum Gene Ther Clin Dev. 2016;27(1):37-48.

44. Boye SL, et al. Impact of Heparan Sulfate Binding on Transduction of Retina by Recombinant Adeno-Associated Virus Vectors. J Virol. 2016;90(8):4215-4231.

45. Choi VW, Asokan A, Haberman RA, Samulski RJ. Production of recombinant adeno-associated viral vectors. Curr Protoc Hum Genet. 2007; Chapter 12:Unit 12.9.

46. Aurnhammer C, et al. Universal real-time PCR for the detection and quantification of adeno-associated virus serotype 2-derived inverted terminal repeat sequences. Hum Gene Ther Methods. 2012;23(1):18-28.

47. Reichel FF, et al. AAV8 Can Induce Innate and Adaptive Immune Response in the Primate Eye. Mol Ther. 2017;25(12):2648-2660.

48. Delyfer MN, et al. Transcriptomic analysis of human retinal detachment reveals both inflammatory response and photoreceptor death. PLoS One. 2011;6(12):e28791.

49. Reichman S, et al. The homeobox gene CHX10/VSX2 regulates RdCVF promoter activity in the inner retina. Hum Mol Genet. 2010;19(2):250-261.

50. Gernoux G, Wilson JM, Mueller C. Regulatory and Exhausted T Cell Responses to AAV Capsid. Hum Gene Ther. 2017;28(4):338-349

51. Takahashi K, et al. Improved Intravitreal AAV-Mediated Inner Retinal Gene Transduction after Surgical Internal Limiting Membrane Peeling in Cynomolgus Monkeys. Mol Ther. 2017;25(1):296-302. 